\title{
Differential forms in the h-topology
}

\author{
Annette Huber and Clemens Jörder
}

\begin{abstract}
We study sheaves of differential forms and their cohomology in the h-topology. This allows one to extend standard results from the case of smooth varieties to the general case. As a first application we explain the case of singularities arising in the Minimal Model Program. As a second application we consider de Rham cohomology.
\end{abstract}

\section{Introduction}

The aim of this note is to propose a new extension of the theory of differential forms to the case of singular varieties in characteristic zero and to illustrate that it has very good properties, unifying a number of ad hoc approaches and allowing a more conceptual understanding of results in the literature.

Differential forms play a key role in the study of local and global properties of manifolds and non-singular algebraic varieties. This principle is confirmed for example by the period isomorphism between algebraic de Rham cohomology and singular cohomology, or the classification of singularities arising from the Minimal Model Program in terms of extension properties of differential forms of top degree.

It is well known that the theory of Kähler differentials is not well behaved in the singular case. Various competing generalizations have been introduced. In each case the definition can be traced back to the non-singular case:

- Kähler differential forms $\Omega_{X}^{p}$ on $X$ and their torsion-free counterpart $\Omega_{X}^{p} /$ tor are obtained as quotients of $\Omega_{Y}^{p}$ for an ambient non-singular space $Y \supset X$.

- Differential forms of first kind on an irreducible variety $X$ are differential forms on a $\log$ resolution $Y \rightarrow X$ (see $[\mathrm{SvS} 85,(1.2)]$ ).

- Reflexive differential forms $\Omega_{X}^{[p]}$ on a normal variety $X$ are differential forms on the regular locus $X^{\text {reg }}$ (see [Kni73], [LW09], [GKKP11])

- Using simplicial hyperresolutions Du Bois [DuB81] defines complexes of coherent sheaves and in this way "localizes Hodge theory".

The purpose of this paper is to introduce a new competitor to this field: h-differential forms $\Omega_{\mathrm{h}}^{p}$. We give three characterizations of very different flavor.

Received 18 June 2013, accepted in final form 30 April 2014.

2010 Mathematics Subject Classification 14F10 (primary), 14F05, 14J17, 14F40 (secondary)

Keywords: differential forms, $h$-topology, klt-spaces, Du Bois complex, de Rham cohomology

This journal is (C) Foundation Compositio Mathematica 2014. This article is distributed with Open Access under the terms of the Creative Commons Attribution Non-Commercial License, which permits non-commercial reuse, distribution, and reproduction in any medium, provided that the original work is properly cited. For commercial re-use, please contact the Foundation Compositio Mathematica.

The second author gratefully acknowledges support by the DFG-Forschergruppe 790 "Classification of Algebraic Surfaces and Compact Complex Manifolds" and the Graduiertenkolleg 1821 "Cohomological Methods in Geometry". 


\section{Annette Huber and Clemens Jörder}

(i) They are the outcome of the sheafification of Kähler differential forms with respect to the htopology on the category of schemes introduced by Voevodsky in [Voe96] (see Definition 3.1).

(ii) They have a simple characterization in birational geometry: Given a variety $X$ we choose arbitrary resolutions $X^{\prime} \rightarrow X$ and $\phi: X^{\prime \prime} \rightarrow X^{\prime} \times_{X} X^{\prime}$. Then pulling back yields a bijection between the set of h-differential forms on $X$ and the set of Kähler differential forms on the resolution $X^{\prime}$ such that the two pull-backs to $X^{\prime \prime}$ coincide. In other words (see Remark 3.8),

$$
\Omega_{\mathrm{h}}^{p}(X) \cong\left\{\alpha \in \Omega_{X}^{p}\left(X^{\prime}\right) \mid\left(\operatorname{pr}_{1} \circ \phi\right)^{*} \alpha=\left(\operatorname{pr}_{2} \circ \phi\right)^{*} \alpha\right\} .
$$

(iii) To give an h-differential form on a variety $X$ is equivalent to giving, in a compatible way, a Kähler differential form on $Y$ for any morphism $Y \rightarrow X$ from a non-singular variety to $X$. More precisely, we have the following result.

TheOREM 1 (Section 3). Let $k$ be a field of characteristic zero and let $X$ be a separated scheme of finite type over $k$. Then

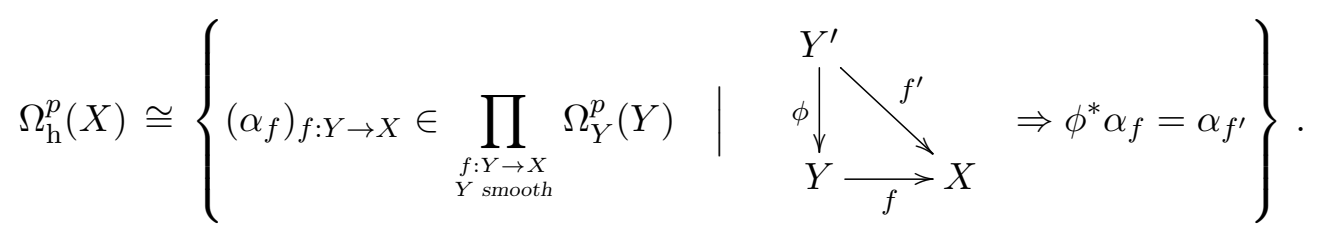

Let us give three reasons why one should consider the h-topology. First, any scheme is hlocally smooth by Hironaka's theorem, an obvious technical advantage.

Second, Kähler differential forms on non-singular schemes turn out to satisfy h-descent (see Theorem 3.6). A variant of this result was first shown by Lee in [Lee09]. An analogous statement has already been observed in the case of the coarser eh-topology by Geisser in [Gei06] and in the even coarser cdh-topology by Cortiñas, Haesemeyer, Walker and Weibel in [CHWW11].

Third, in contrast to the cdh- or eh-topology, all proper surjective morphisms and all flat covers are h-covers. Recall that proper covers in the context of de Rham cohomology were introduced long ago by Deligne in [Del74] in order to extend the period isomorphism to the singular case. We would like to emphasize the flexibility gained by using arbitrary h-covers. In many cases, technical difficulties disappear thanks to the machinery.

These technical advantages allow us to prove invariance of h-differentials for maps with rationally chain connected fibres (Theorem 5.12). Together with the extension theorem of Greb, Kebekus, Kovács and Peternell in [GKKP11], this implies the following result for varieties whose singularities arise in the Minimal Model Program.

TheOREM 2 (Section 5). On a klt base space, we have $\Omega_{\mathrm{h}}^{p}(X)=\Omega_{X}^{[p]}(X)$.

The special case of normal toric varieties has already been proved in [CHWW09, Theorem 4.1]; see Remark 5.5 for more details. By Theorem 2 we obtain a more conceptual explanation for the existence of pull-back maps for reflexive differential forms on klt base spaces (see Corollary 5.6). This is the main result in [Keb13]. On the other hand, [Keb13] and Theorem 1 together imply Theorem 2.

Let us now turn to the study of the cohomology of h-differentials. The natural notion is cohomology of h-sheaves. This does not change anything in the smooth case.

TheOREm 3 (Section 6 ). On a smooth variety, we have $H_{\mathrm{h}}^{i}\left(X, \Omega_{\mathrm{h}}^{p}\right)=H_{\mathrm{Zar}}^{i}\left(X, \Omega_{X}^{p}\right)$. 


\section{DIFFERENTIAL FORMS IN THE h-TOPOLOGY}

The main computational tool, the blow-up sequence, allows one to easily compute the cohomology of singular varieties from the smooth case.

Subsequently we will analyse in more detail the relation between the h-topology on the category of schemes over a scheme $X$ and the Zariski topology on $X$. This is useful in two ways.

First, as already realized by Lee ([Lee09]), the new point of view offers a new perspective on the Du Bois complex of a variety. It turns out to be the derived push-forward of the h-differential forms considered as a complex of sheaves in the h-topology (Theorem 7.12). To illustrate the advantage of our language we subsequently deduce a number of well-known properties of the Du Bois complex. In contrast to [DuB81] and [GNPP88], we avoid any use of simplicial or cubic hyperresolutions in the construction.

Analogous results in terms of the cdh-topology were also shown by Cortiñas, Haesemeyer, Schlichting, Walker and Weibel as a byproduct of their work on homotopy invariance of algebraic $K$-theory; see their series of papers [CHSW08], [CHW08], [CHWW09], [CHWW10], [CHWW11], [CHWW13].

This leads to the last application. Hypercohomology of the complex of h-sheaves $\Omega_{\mathrm{h}}^{p}$ gives a simple definition of algebraic de Rham cohomology. It agrees with the other definitions in the literature. The question to what extent the analytified sheaves of h-differential forms resolve the sheaf of locally constant functions is treated in the dissertation [Jör14] of the second author.

As an application of our techniques, we use the machinery of h-differential forms to construct the relative de Rham cohomology associated with a closed subscheme $Z \subset X$. It is the counterpart of relative singular cohomology and is needed in the study of the period isomorphism and the period numbers of a general variety, for example, in the work of Kontsevich and Zagier on periods [KZ02]; see also [HM11]. Its existence and properties have been known to experts, but we are not aware of a good reference. The obvious definition via the shift of a reduced cone of hyperresolutions makes establishing the properties very difficult. It is not even clear to us how to write down the Künneth morphism. One possible approach is by using Saito's theory of Hodge modules, another is by making systematic use of the de Rham realization of triangulated motives (see [Hub00] and [Hub04], or [LW09]). Using h-differentials we are able to write down a simple definition and give straightforward algebraic proofs for basic features such as long exact sequences associated with triples, excision and the Künneth formula (Subsection 7.3).

Beilinson in his approach to $p$-adic Hodge theory (see [Bei12]) also uses the h-topology in order to study the de Rham complex. His construction is a lot more subtle. He is working over the ring of integers of a local field of mixed characteristic, that is, with integral (or rather $p$-adic) coefficients. Moreover, his $\mathcal{A}_{\mathrm{dR}}$ is a projective system of complexes of h-sheaves which is built on Illusie's cotangent complex instead of the cotangent space. We have not tried to work out the explicit relation to $\Omega_{\mathrm{h}}^{*}$ on the generic fibre.

Using the h-topology in the context of de Rham cohomology is quite natural: the de Rham complex is a homotopy invariant complex of sheaves of $\mathbb{Q}$-vector spaces with transfers. From the general motivic machinery we learn that its cohomology can be equivalently treated in the h-topology without transfers. One point we want to demonstrate in our paper is that the hsheafification of the individual $\Omega^{p}$ is also very useful.

The present paper concentrates on differential forms rather than developing a full-blown six-functor formalism for $\mathcal{O}$-modules in the h-topology. We have refrained from dealing with differential forms with log poles or with twists by line bundles. What is also missing is a discussion of Grothendieck duality where differential forms also play a key role. We work in characteristic 


\section{Annette Huber and Clemens Jörder}

zero throughout and hope that a modified definition would also work in positive characteristic. We hope that these aspects will be developed in the future.

\section{Outline of the paper}

The paper consists of two parts. Sections 2 through 5 discuss sheaves of differential forms in various topologies. Sections 6 and 7 deal with cohomology groups and objects in the derived category.

The goal of Sections 2 and 3 is to establish h-descent for differential forms. Section 2 recalls the results of Geisser [Gei06] in his eh-topology and establishes additional properties of differentials forms in the eh-topology. Section 3 reviews Voevodsky's h-topology and deduces Theorem 1 mentioned above.

In Section 4, we make the definition of $\Omega_{\mathrm{h}}^{p}(X)$ explicit in a number of cases: for $p=0$, $p=\operatorname{dim} X$ or when $X$ has special types of singularities.

Section 5 treats the case of klt-singularities. Theorem 2 is deduced from the key result on the invariance of h-differentials for maps with rationally chain connected fibres.

We then turn in Section 6 to the cohomology of sheaves in the h-topology in general and to the cohomology of h-differential forms.

Finally, in Section 7, we consider the de Rham complex of h-differential forms. We recover the $\mathrm{Du}$ Bois complex in terms of h-differential forms. The section concludes with a simple description of relative algebraic de Rham cohomology in terms of h-differential forms.

\section{Setting and Notation}

We fix a field $k$ of characteristic zero. By scheme we mean a separated scheme of finite type over $k$. By variety we mean a reduced separated scheme of finite type over $k$. We denote by Sch, Var and Sm the categories of $k$-schemes, $k$-varieties and smooth $k$-varieties, respectively.

A resolution of an irreducible variety $X$ is a proper birational morphism $X^{\prime} \rightarrow X$ from a smooth variety $X^{\prime}$ to $X$. A resolution of a variety $X$ is a morphism $X^{\prime} \rightarrow X$ where $X^{\prime}$ is the disjoint union of resolutions of the irreducible components of $X$.

If $t$ is a Grothendieck topology, we denote by $\mathrm{Sch}_{t}, \operatorname{Var}_{t}, \mathrm{Sm}_{t}$ the site defined by $t$ and by $\left(\mathrm{Sch}_{t}\right)^{\sim},\left(\operatorname{Var}_{t}\right)^{\sim}$ and $\left(\mathrm{Sm}_{t}\right)^{\sim}$ the topos of sheaves of sets on $\mathrm{Sch}_{t}, \operatorname{Var}_{t}$ and $\mathrm{Sm}_{t}$, respectively. We are going to consider the cases Zar (Zariski topology), et (étale topology), eh (étale h-topology; see Definition 2.1) and h (h-topology; see Definition 3.1).

If $\mathcal{F}$ is a $t$-sheaf of abelian groups in some Grothendieck topology on Sch and $X \in \mathrm{Sch}$, then we write $H_{t}^{i}(X, \mathcal{F})$ for the $i$ th derived functor of $\Gamma(X, \cdot)$ evaluated on $\mathcal{F}$.

Definition 1.1. Let $X \in \mathrm{Sch}$, and let $t$ be some Grothendieck topology on Sch. We write $\mathbb{Z}_{t}(X)$ for the $t$-sheafification of the presheaf

$$
T \mapsto \mathbb{Z}[X(T)],
$$

where $\mathbb{Z}[S]$ denotes the free abelian group generated by $S$.

Recall that

$$
H_{t}^{i}(X, \mathcal{F})=\operatorname{Ext}^{i}\left(\mathbb{Z}_{t}(X), \mathcal{F}\right)
$$

for all t-sheaves $\mathcal{F}$. 


\section{DifFERENTIAL FORMS IN THE h-TOPOLOGY}

For a $k$-scheme $X$ let $\Omega_{X}^{1}$ be the Zariski sheaf of $k$-linear Kähler differentials on $X$. For $p \geqslant 0$, let $\Omega_{X}^{p}$ be the $p$-th exterior power of $\Omega_{X}^{1}$ in the category of $\mathcal{O}_{X}$-modules. We denote by $\Omega^{p}$ the sheaf

$$
X \mapsto \Omega_{X}^{p}(X)
$$

on the big Zariski site on $\operatorname{Sch}_{k}$. The usual differential

$$
d: \Omega^{p} \rightarrow \Omega^{p+1}
$$

turns it into a differential graded algebra $\Omega^{\bullet}$. If $t$ is another topology on Sch, we denote by $\Omega_{t}^{\bullet}$ the sheafification in the $t$-topology.

We are also going to consider Zariski differentials, which are studied for example in [Kni73] and [GKKP11]. We follow the notation of the second reference.

Definition 1.2. Let $X$ be a normal variety, and let $j: X^{\text {reg }} \rightarrow X$ be the inclusion of the regular locus. We call $\Omega_{X}^{[p]}=j_{*} \Omega_{X^{\text {reg }}}^{p}$ the sheaf of Zariski differentials on $X$ or sheaf of reflexive differentials.

\section{Differential forms in the eh-topology}

We review the eh-topology introduced by Geisser in [Gei06]. It is a twin of the cdh-topology introduced by Voevodsky in [Voe96]. The relation of the eh-topology to the étale topology is the same as the relation of the Nisnevich topology to the cdh-topology. For our purposes it makes no difference which is used. We consider differential forms in the eh-topology.

Definition 2.1 [Gei06, Definition 2.1]. The eh-topology on the category Sch of separated schemes of finite type over $k$ is the Grothendieck topology generated by the following coverings:

- étale coverings

- abstract blow-ups: assume that we have a Cartesian square

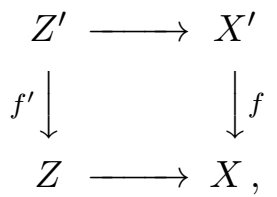

where $f$ is proper, $Z \subset X$ is a closed subscheme and $f$ induces an isomorphism $X^{\prime} \backslash Z^{\prime} \rightarrow$ $X \backslash Z$; then $\left(X^{\prime} \rightarrow X, Z \rightarrow X\right)$ is a covering.

Example 2.2. (i) Let $f: X_{\text {red }} \subset X$ be the reduction. Then $f$ is an eh-cover (with $X^{\prime}=\emptyset$, $\left.Z=X_{\text {red }}\right)$. Hence every scheme is eh-locally reduced.

(ii) Every proper morphism $X^{\prime} \rightarrow X$ such that for every $x \in X$ there is a point in $p^{-1}(x)$ with the same residue field as $x$ is an eh-covering by [Gei06, Lemma 2.2]. A special case is a blow-up of a smooth variety in a smooth centre.

Definition 2.3. A covering as in Example 2.2 (ii) is called a proper eh-covering.

Construction 2.4. By resolution of singularities, every $X \in$ Sch has a proper eh-cover $\left\{\tilde{X}_{i} \rightarrow\right.$ $X\}_{i \in I}$ by smooth varieties $\tilde{X}_{i}$. We spell out the algorithm.

(i) The scheme $X \in$ Sch has a proper eh-cover $X$ by $X_{\text {red }}$. 


\section{Annette Huber And Clemens Jörder}

(ii) Let $X=\bigcup_{i=1}^{n} X_{i} \in \operatorname{Var}$ with $X_{i}$ irreducible. Then

$$
\left(\coprod_{i=1}^{n} X_{i} \rightarrow X, \bigcup_{i<j} X_{i} \cap X_{j}\right)
$$

is an abstract blow-up and hence a proper eh-cover.

(iii) Let $X$ be an irreducible variety. By resolution of singularities there is a birational proper map $\pi: \tilde{X} \rightarrow X$ with $\tilde{X}$ smooth. Let $Z \subset X$ be the image in $X$ of the exceptional locus of $\pi$. Then $\{\tilde{X}, Z\}$ is an abstract blow-up and hence a proper eh-cover.

(iv) Let $Z$ be as in the last step. By induction on the dimension, there is a proper eh-cover of $Z$ by smooth varieties.

Definition 2.5. For $p \geqslant 0$, let $\Omega_{\mathrm{eh}}^{p}$ be the eh-sheafification of the presheaf

$$
X \mapsto \Omega^{p}(X)
$$

on the category Sch. We call the elements of $\Omega_{\mathrm{eh}}^{p}(X)$ eh-differentials on $X$.

Proposition 2.6 [Gei06, Theorem 4.7]. Let $X$ be a smooth variety. Then

$$
\Omega^{p}(X)=\Omega_{\mathrm{eh}}^{p}(X) .
$$

Remark 2.7. The proof of Proposition 2.6 in [Gei06, Theorem 4.7] appears rather technical. The main mathematical content of Proposition 2.6 is that $\Omega^{p}$ satisfies the sheaf property for ehcovers of a smooth variety by a smooth variety. This fact might already be known to the reader in special cases such as an étale cover of a smooth variety (see [Mil80, III Proposition 3.7]), or a blowup $X_{Z} \rightarrow X$ of a smooth variety along a smooth subvariety (observe that $\Omega^{p}\left(X_{Z}\right) \cong \Omega^{p}(X)$ by [Gro85, Chapitre IV, Théorème 1.2.1] in this case). The general case follows from these by resolution of singularities.

In order to connect our results with an alternative version considered elsewhere in the literature, we record another comparison result.

COROLlary 2.8. Let $\Omega_{\mathrm{cdh}}^{p}$ be the sheafification of $\Omega^{p}$ with respect to the cdh-topology (generated by abstract blow-ups and Nisnevich covers). Then for any $X \in \mathrm{Sch}$, we have $\Omega_{\mathrm{cdh}}^{p}(X)=\Omega_{\mathrm{eh}}^{p}(X)$.

Proof. Note that the cdh-topology is coarser than the eh-topology but still contains all abstract blow-ups. Thus Construction 2.4 shows that all schemes are cdh-locally smooth. Hence it suffices to consider the case $X$ smooth.

We combine the comparison theorem for the eh-topology with its analogue in the cdh-topology in [CHWW11, Lemma 2.9].

We now establish properties of eh-differentials needed later on.

Lemma 2.9. Let $f: X \rightarrow Y$ be a dominant morphism. Then $\Omega_{\mathrm{eh}}^{p}(Y) \hookrightarrow \Omega_{\mathrm{eh}}^{p}(X)$ is injective.

Proof. First assume that $Y$ and $X$ are smooth and irreducible. By Proposition 2.6 we have to consider $\Omega^{p}$. As $\Omega^{p}$ is a vector bundle on $X$, we have $\Omega^{p}(X) \subset \Omega^{p}(k(X) / k)$. As $f$ is a dominant morphism between irreducible schemes of characteristic zero, we have $\Omega^{p}(k(Y) / k) \subset \Omega^{p}(k(X) / k)$. This settles the smooth case.

In general, we may assume that $Y=Y_{\text {red }}$. Let $\alpha \in \Omega_{\text {eh }}^{p}(Y)$ and write $\left.\alpha\right|_{T}$ for the pull-back of $\alpha$ by a map $T \rightarrow Y$. We assume that $\left.\alpha\right|_{X}=0$ and seek to prove that $\alpha=0$. By Construction 2.4 it suffices to show that $\left.\alpha\right|_{T}=0$ for all $T \rightarrow Y$ where $T$ is smooth and irreducible. 
Choose a resolution $S^{\prime} \rightarrow\left(X \times_{Y} T\right)_{\text {red }}$ of singularities and let $S \subset S^{\prime}$ be an irreducible component dominating $T$. Then $\left.\alpha\right|_{X}=0$ implies $\left.\alpha\right|_{S}=0$. This implies $\left.\alpha\right|_{T}=0$ by the smooth case.

Lemma 2.10. Let $X$ be normal and irreducible, let $K / K(X)$ be a Galois extension with Galois group $G$ and let $Y$ be the normalization of $X$ in $K$. Then

$$
\Omega_{\mathrm{eh}}^{p}(X)=\Omega_{\mathrm{eh}}^{p}(Y)^{G}
$$

and $\Omega_{\mathrm{eh}}^{p}$ has descent for $\pi: Y \rightarrow X$, that is, the sequence

$$
0 \rightarrow \Omega^{p}(X) \rightarrow \Omega_{\mathrm{eh}}^{p}(Y) \stackrel{p r_{1}^{*}-p r_{2}^{*}}{\longrightarrow} \Omega_{\mathrm{eh}}\left(Y \times_{X} Y\right)
$$

is exact.

Proof. If $X$ is smooth and $Y \rightarrow X$ is étale, then $Y$ is also smooth and the lemma holds because $\Omega^{p}$ has étale descent. In the general case, let $U \subset X$ be a smooth open subscheme over which $\pi$ is étale. We get a commutative diagram

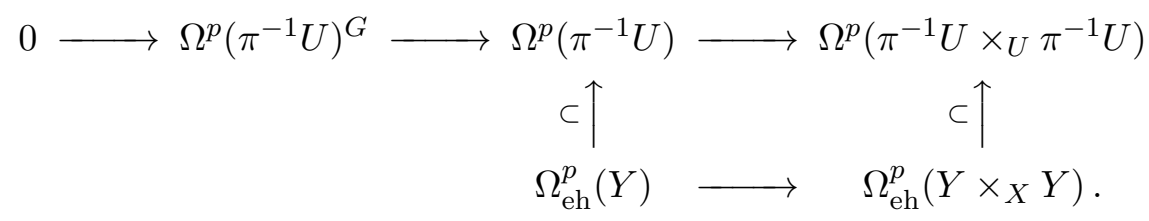

The top line is exact by the special case, so that

$$
\Omega^{p}\left(\pi^{-1} U\right)^{G} \cap \Omega_{\mathrm{eh}}^{p}(Y)=\Omega_{\mathrm{eh}}^{p}(Y)^{G}
$$

is the kernel $\Omega_{\mathrm{eh}}^{p}(X)$ of the lower line.

\section{Differential forms in the h-topology}

We first review the definition of the h-topology and its properties from [Voe96]. We then study the case of the sheaf of differential forms.

Definition 3.1 [Voe96, Definitions 3.1.1 and 3.1.2]. A morphism $p: \tilde{X} \rightarrow X$ in Sch is called a topological epimorphism if $p$ is surjective and the Zariski topology of $X$ is the quotient topology of the Zariski topology of $\tilde{X}$. It is called a universal topological epimorphism if its base change by any $f: Z \rightarrow X$ in Sch is also a topological epimorphism.

The h-topology on Sch is the Grothendieck topology whose covering systems $\left\{p_{i}: U_{i} \rightarrow X\right\}_{i \in I}$ are such that

$$
\coprod_{i \in I} p_{i}: \coprod_{i \in I} U_{i} \rightarrow X
$$

is a universal topological epimorphism.

Example 3.2 [Voe96, Section 3.1]. The following are h-covers:

(i) flat covers;

(ii) proper surjective morphisms;

(iii) quotients by the operation of a finite group.

In particular, eh-covers are h-covers. The case of abstract blow-ups is particularly useful. 


\section{Annette Huber and Clemens Jörder}

Proposition 3.3 (Blow-up square). Let $\left(X^{\prime}, Z\right)$ be an abstract blow-up of $X$ with $E=X^{\prime} \times_{X} Z$. Let $\mathcal{F}$ be an h-sheaf. Then the blow-up square

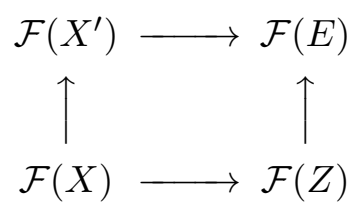

is Cartesian.

Proof. We may assume that all schemes are reduced. Then the statement is equivalent to the sheaf condition for the h-cover $\left(X^{\prime} \rightarrow X, Z \rightarrow X\right)$ using the fact that

$$
\left(\operatorname{diag}: X^{\prime} \rightarrow X^{\prime} \times_{X} X^{\prime}, E \times_{Z} E \subset X^{\prime} \times_{X} X^{\prime}\right)
$$

is an h-cover.

Proposition 3.4. Let $\mathfrak{U}=\left\{p_{i}: U_{i} \rightarrow X\right\}_{i \in I}$ be an h-covering in Sch. Then there exists a refinement such that the index set $I^{\prime}$ is finite and $p_{i}^{\prime}$ factors as

$$
U_{i}^{\prime} \stackrel{\iota_{i}}{\rightarrow} \bar{U}^{\prime} \stackrel{f}{\rightarrow} X^{\prime} \stackrel{\pi}{\rightarrow} X
$$

where $\left\{\iota_{i}: U_{i}^{\prime} \rightarrow \bar{U}^{\prime}\right\}_{i \in I}$ is an open covering in the Zariski topology, $f$ is finite surjective and $\pi$ is a proper eh-covering by a smooth variety.

Proof. By Construction 2.4 choose a proper eh-cover $Y \rightarrow X$ with $Y$ smooth and consider the pull-back $\mathfrak{U}^{\prime}$ of $\mathfrak{U}$ to $Y$. By [Voe96, Theorem 3.1.9] we may assume that $\mathfrak{U}$ has a refinement in normal form, that is, $\left\{U_{i}^{\prime} \rightarrow \bar{U}^{\prime} \rightarrow Y^{\prime} \rightarrow Y\right\}_{i \in I}$ with $Y^{\prime} \rightarrow Y$ a blow-up of $Y$ in a closed subvariety, $\bar{U}^{\prime} \rightarrow Y^{\prime}$ finite surjective and the system of $\iota_{i}$ a finite open cover of $\bar{U}^{\prime}$. By blowing up further we may assume that $Y^{\prime}$ is smooth and $Y^{\prime} \rightarrow Y$ is a sequence of blow-ups in smooth centres. Then $Y^{\prime} \rightarrow Y$ is a proper eh-covering by Example 2.2 (ii). We choose $\pi: X^{\prime}=Y^{\prime} \rightarrow Y \rightarrow X$.

DeFinition 3.5. Let $\Omega_{h}^{p}$ be the sheafification of $\Omega^{p}$ (or equivalently $\Omega_{\mathrm{eh}}^{p}$ ) in the h-topology. Elements of $\Omega_{h}^{p}(X)$ are called h-differentials on $X$.

Theorem 3.6 (h-descent). The presheaf $\Omega_{\mathrm{eh}}^{p}$ on Sch has h-descent, that is,

$$
\Omega_{\mathrm{h}}^{p}(X)=\Omega_{\mathrm{eh}}^{p}(X)
$$

for all $X \in$ Sch. In particular,

$$
\Omega_{\mathrm{h}}^{p}(X)=\Omega^{p}(X)
$$

for all $X \in \mathrm{Sm}$.

Proof. The second assertion follows from the first by Proposition 2.6.

We need to check that for all $X$ in Sch, the morphism

$$
\phi_{X}: \Omega_{\mathrm{eh}}^{p}(X) \rightarrow \lim _{\mathfrak{U}} \check{H}^{0}\left(\mathfrak{U}, \Omega_{\mathrm{eh}}^{p}\right),
$$

where $\mathfrak{U}$ runs through all h-covers of $X$, is an isomorphism. Let $X$ be in Sch and let $\mathfrak{U}$ be an h-cover of $X$. It suffices to verify the sheaf condition for $\Omega_{\mathrm{eh}}^{p}$ on a refinement of $\mathfrak{U}$. We claim that the sheaf condition is satisfied in three special cases:

(i) when $\mathfrak{U}$ is a Zariski cover;

(ii) when $\mathfrak{U}=\left\{X^{\prime} \rightarrow X\right\}$ is a proper eh-cover; 


\section{DiFFERENTIAL FORMS IN THE h-TOPOLOGY}

(iii) when $\mathfrak{U}=\left\{X^{\prime} \rightarrow X\right\}$ is a finite and surjective map of irreducible normal varieties such that $k\left(X^{\prime}\right) / k(X)$ is Galois.

The first two cases hold because they are eh-covers. In the third case, the sheaf condition holds by Lemma 2.10 .

Now let $\mathfrak{U}$ be a general h-covering of $X$. After refining it, we may assume by Proposition 3.4 that it is of the form $\left\{U_{i} \rightarrow \bar{U} \rightarrow X^{\prime} \rightarrow X\right\}_{i \in I}$ with $X^{\prime} \rightarrow X$ a proper eh-cover with $X^{\prime}$ smooth, $\bar{U} \rightarrow X$ finite and surjective and $\left\{U_{i}\right\}_{i \in I}$ an open cover of $\bar{U}$. For every connected component $X_{j}^{\prime}$ of $X^{\prime}$ choose an irreducible component $\bar{U}_{j}$ of $\bar{U}$ mapping surjectively to $X_{j}^{\prime}$. We refine the cover by replacing $\bar{U}$ by the disjoint union of the normalizations of $\bar{U}_{j}$ in the normal hull of $k\left(\bar{U}_{j}\right) / k\left(X_{j}^{\prime}\right)$.

The sheaf condition is satisfied in the three intermediate steps. In particular, or by Lemma 2.9, we have injections

$$
\Omega_{\mathrm{eh}}^{p}(X) \hookrightarrow \Omega_{\mathrm{eh}}^{p}\left(X^{\prime}\right) \hookrightarrow \Omega_{\mathrm{eh}}^{p}(\bar{U}) \hookrightarrow \prod_{i \in I} \Omega_{\mathrm{eh}}^{p}\left(U_{i}\right) .
$$

Moreover, $\bar{U} \times_{X} \bar{U} \rightarrow X^{\prime} \times_{X} X^{\prime}$ is proper and surjective, hence by Lemma 2.9

$$
\Omega_{\mathrm{eh}}^{p}\left(X^{\prime} \times_{X} X^{\prime}\right) \hookrightarrow \Omega_{\mathrm{eh}}^{p}\left(\bar{U} \times_{X} \bar{U}\right) .
$$

A little diagram chase then allows us to conclude from the sheaf conditions for $X^{\prime} \rightarrow X$ and $\bar{U} \rightarrow X^{\prime}$ that the sheaf condition is satisfied for $\bar{U} \rightarrow X$. We prove the theorem by repeating the argument with $\left\{U_{i} \rightarrow \bar{U}\right\}_{i \in I}$.

Remark 3.7. One of the main results of Lee in [Lee09] is the fact that $\Omega^{p}$ is a sheaf on Sm equipped with the $h$-topology (loc. cit. Proposition 4.2). This seems basically the same as the above Theorem 3.6, though we did not check the details. His proof is different and made technically more complicated by the fact that $\mathrm{Sm}$ is not closed under fibre products.

Remark 3.8. Note that the proof contains a simple formula for $\Omega_{h}^{p}(X)$ : Choose an h-cover $X^{\prime} \rightarrow X$ with $X^{\prime}$ smooth. Choose an h-cover $X^{\prime \prime} \rightarrow X^{\prime} \times_{X} X^{\prime}$ with $X^{\prime \prime}$ smooth. Then

$$
0 \rightarrow \Omega_{\mathrm{h}}^{p}(X) \rightarrow \Omega^{p}\left(X^{\prime}\right) \rightarrow \Omega^{p}\left(X^{\prime \prime}\right)
$$

is exact.

COROLlary 3.9. Theorem 1 of the introduction holds.

Proof. We call a family $\left(\alpha_{f}\right)_{f}$ as in Theorem 1 a compatible family. By Theorem 3.6, any $\beta \in$ $\Omega_{\mathrm{h}}^{p}(X)$ determines a compatible family $\beta_{f}=f^{*}(\beta) \in \Omega^{p}(Y)=\Omega_{\mathrm{h}}^{p}(Y)$.

Conversely, let us choose maps $X^{\prime} \stackrel{g}{\rightarrow} X$ and $X^{\prime \prime} \stackrel{h}{\rightarrow} X^{\prime} \times_{X} X^{\prime} \stackrel{i}{\rightarrow} X$ as in Remark 3.8. For any compatible family $\left(\alpha_{f}\right)_{f}$ we have $\left(\operatorname{pr}_{1} \circ h\right)^{*} \alpha_{g}=\alpha_{i \circ h}=\left(\operatorname{pr}_{2} \circ h\right)^{*} \alpha_{g}$, so that there exists a unique $\beta \in \Omega_{\mathrm{h}}^{p}(X)$ such that $\alpha_{g}=g^{*} \beta$.

It remains to show that $f^{*}(\beta)=\alpha_{f}$ for any morphism $f: Y \rightarrow X$ from a non-singular variety $Y$ to $X$. Let $p: Y^{\prime} \rightarrow\left(Y \times_{X} X^{\prime}\right)_{\text {red }}$ be a resolution and denote the map to $X$ by $j: Y^{\prime} \rightarrow X$. Then the claim follows from

$$
\left(\operatorname{pr}_{Y} \circ p\right)^{*} \alpha_{f}=\alpha_{j}=\left(\operatorname{pr}_{X^{\prime}} \circ p\right)^{*} \alpha_{g}=\left(\operatorname{pr}_{X^{\prime}} \circ p\right)^{*} g^{*} \beta=\left(\operatorname{pr}_{Y} \circ p\right)^{*} f^{*} \beta
$$

and the injectivity of $\left(\operatorname{pr}_{Y} \circ p\right)^{*}: \Omega_{\mathrm{h}}^{p}(Y) \rightarrow \Omega_{\mathrm{h}}^{p}\left(Y^{\prime}\right)$.

The theorem gives a more conceptual proof for the result of Lecomte and Wach on the existence of transfers. 


\section{Annette Huber and Clemens Jörder}

Corollary 3.10 ([LW09]). The presheaf $\Omega^{p}$ is an (étale) sheaf with transfers on Sm.

Proof. The presheaf $\Omega_{\mathrm{h}}^{p}$ is by definition an h-sheaf. By [Scho12, Proposition 2.2] it has a canonical transfer structure. By Theorem 3.6, we have $\Omega_{h}^{p}(X)=\Omega^{p}(X)$ for smooth $X$.

Remark 3.11. Having achieved the identification of $\Omega_{\mathrm{eh}}^{p}$ and $\Omega_{\mathrm{h}}^{p}$, the results of the previous section trivially apply to $\Omega_{\mathrm{h}}^{p}$ as well. Moreover, by Corollary 2.8 we can also identify $\Omega_{\mathrm{h}}^{p}$ with $\Omega_{\mathrm{cdh}}^{p}$. Hence all results in the series of papers by Cortiñas, Haesemeyer, Schlichting, Walker and Weibel ([CHSW08], [CHW08], [CHWW09], [CHWW10], [CHWW11], [CHWW13]) on $\Omega_{\mathrm{cdh}}^{p}$ can be read as results on $\Omega_{\mathrm{h}}^{p}$. For example, [CHWW09, Theorem 4.1] in degree zero and Corollary 2.8 give a proof of our Remark 5.5.

\section{First Properties and Examples}

In this section we gather some facts about h-differential forms when considered as sheaves in the Zariski topology. To this end, let us introduce the following notation.

Definition 4.1. Let $\mathcal{F}$ be an h-sheaf on Sch. We write $\left.\mathcal{F}\right|_{X}$ for the Zariski sheaf $U \mapsto \mathcal{F}(U)$ for $U \subset X$ open.

The basic properties of the resulting sheaves $\left.\Omega_{\mathrm{h}}^{p}\right|_{X}$ and their relation to other sheaves of differential forms are summarized below.

Proposition 4.2 (Properties of $\left.\Omega_{\mathrm{h}}^{p}\right|_{X}$ ). The sheaves $\left.\Omega_{\mathrm{h}}^{p}\right|_{X}$ satisfy the following:

(i) The sheaf $\left.\Omega_{\mathrm{h}}^{p}\right|_{X}$ is a torsion-free coherent sheaf of $\mathcal{O}_{X}$-modules.

(ii) If $X$ is smooth, then $\left.\Omega_{\mathrm{h}}^{p}\right|_{X}=\Omega_{X}^{p}$.

(iii) If $r: X_{\text {red }} \rightarrow X$ is the reduction, then $\left.r_{*} \Omega_{\mathrm{h}}^{p}\right|_{X_{\mathrm{red}}}=\left.\Omega_{\mathrm{h}}^{p}\right|_{X}$.

(iv) If $X$ is reduced, there exists a natural inclusion $\Omega_{X}^{p} /$ torsion $\left.\subset \Omega_{\mathrm{h}}^{p}\right|_{X}$.

(v) If $X$ is normal, there exists a natural inclusion $\left.\Omega_{\mathrm{h}}^{p}\right|_{X} \subset \Omega_{X}^{[p]}$.

(vi) For any $p>\operatorname{dim}(X)$, we have $\left.\Omega_{\mathrm{h}}^{p}\right|_{X}=0$.

Proof. Recall that we have $\Omega_{\mathrm{eh}}^{p}=\Omega_{\mathrm{h}}^{p}$ by Theorem 3.6.

Let us choose $X^{\prime}$ and $X^{\prime \prime}$ as in Remark 3.8 and denote the maps to $X$ by $\pi^{\prime}: X^{\prime} \rightarrow X$ and $\pi^{\prime \prime}: X^{\prime \prime} \rightarrow X$. We may assume that both $X^{\prime}$ and $X^{\prime \prime}$ are proper over $X$. Then $\left.\Omega_{\mathrm{h}}^{p}\right|_{X}$ is the kernel of a morphism $\pi_{*}^{\prime} \Omega_{X^{\prime}}^{p} \rightarrow \pi_{*}^{\prime \prime} \Omega_{X^{\prime \prime}}^{p}$ between coherent sheaves which gives the coherence in item (i). It is torsion-free by Lemma 2.9. Item (ii) follows from the sheafification of Theorem 3.6. Item (iii) follows from the h-sheaf condition for the cover $X^{\text {red }} \rightarrow X$.

For item (iv) note that by item (i) the natural map $\left.\Omega_{X}^{p} \rightarrow \Omega_{\mathrm{h}}^{p}\right|_{X}$ induces a map $\Omega_{X}^{p} /$ torsion $\rightarrow$ $\left.\Omega_{\mathrm{h}}^{p}\right|_{X}$. We have to check that it is injective. Let $U \subset X_{\text {red }}=X$ be the smooth locus. By torsionfreeness, $\left.\left.\Omega_{\mathrm{h}}^{p}\right|_{X} \subset j_{*} \Omega_{\mathrm{h}}^{p}\right|_{U}=j_{*} \Omega_{U}^{p}$. On the other hand, $\Omega_{X_{\text {red }}}^{p}$ /torsion $\subset j_{*} \Omega_{U}^{p}$ because both agree on $U$.

Let $X$ be normal and let $U$ be the smooth locus. By Lemma 2.9 and Definition 1.2 the restriction map $\Omega_{\mathrm{h}}^{p}(X) \rightarrow \Omega_{\mathrm{h}}^{p}(U)=\Omega^{p}(U)=\Omega^{[p]}(X)$ is injective. Hence item (v) holds.

Finally, for item (vi), by item (iii) we may assume that $X$ is reduced, and by item (i) we may restrict to an open subset where it is smooth. By item (ii), the vanishing follows from the vanishing for Kähler differentials. 


\section{DiFFERENTIAL FORMS IN THE h-TOPOLOGY}

We now turn to the study of the two extreme cases $p=0$ and $p=\operatorname{dim}(X)$. First, let us consider the case $p=0$ and observe that $\mathcal{O}_{\mathrm{h}}=\Omega_{\mathrm{h}}^{0}$.

Definition 4.3 [Tra70]. Let $X$ be a variety with total ring of fractions $K(X)$. The seminormalization $X^{\mathrm{sn}}$ of $X$ is the maximal finite cover $\pi: X^{\mathrm{sn}} \rightarrow X$ which is bijective on points and induces an isomorphism on residue fields. If $X$ is not reduced, we define $X^{\mathrm{sn}}$ as the seminormalization of the reduction of $X$.

A variety is called semi-normal if it agrees with its semi-normalization.

Example 4.4. (i) Normal varieties are semi-normal.

(ii) The cuspidal curve with equation $y^{2}=x^{3}$ has as semi-normalization the affine line.

(iii) The nodal curve with equation $y^{2}=x^{2}(x-1)$ is semi-normal.

Proposition 4.5. Let $X \in$ Sch. Then

$$
\mathcal{O}_{\mathrm{h}}(X)=\mathcal{O}\left(X^{\mathrm{sn}}\right) .
$$

In particular, $\mathcal{O}_{\mathrm{h}}(X)=\mathcal{O}(X)$ if $X$ is semi-normal.

Proof. We write $\tilde{X}$ for the presheaf $T \mapsto X(T)$ so that $\mathcal{O}=\tilde{\mathbb{A}}^{1}$. As in [Voe96, Section 3.2], we write $L(X)$ for the h-sheaf associated with $\tilde{X}$, which means that $\mathcal{O}_{\mathrm{h}}=L\left(\mathbb{A}^{1}\right)$. By the universal property of sheafification,

$$
\mathcal{O}_{\mathrm{h}}(X)=\operatorname{Mor}_{L(\mathrm{Sch})}\left(L(X), L\left(\mathbb{A}^{1}\right)\right),
$$

where $L(\mathrm{Sch})$ is the category of representable h-sheaves. Moreover, [Voe96, Proposition 3.2.10] asserts that

$$
\mathcal{O}_{\mathrm{h}}(X)=\operatorname{Mor}_{L(\operatorname{Sch})}\left(L(X), L\left(\mathbb{A}^{1}\right)\right)=\operatorname{Mor}_{\mathrm{Sch}}\left(R L(X), \mathbb{A}^{1}\right)=\mathcal{O}(R L(X)),
$$

where $R L(X)=X^{\mathrm{sn}}$ is the semi-normalization of $X$.

We now turn to the other extreme case, $p=\operatorname{dim} X$.

Proposition 4.6. Let $X$ be a variety of dimension $d$ and let $\pi: \tilde{X} \rightarrow X$ be a resolution, that is, a proper birational morphism with $\tilde{X}$ smooth. Then $\left.\Omega_{\mathrm{h}}^{d}\right|_{X}=\pi_{*} \Omega_{\tilde{X}}^{d}$.

Proof. Let $E \subset \tilde{X}$ be the reduced exceptional locus of $\pi$, and let $i: Z:=\pi(E) \rightarrow X$ be the inclusion map of its image in $X$. The blow-up sequence

$$
\left.\left.\left.\left.0 \rightarrow \Omega_{\mathrm{h}}^{d}\right|_{X} \rightarrow \pi_{*} \Omega_{\mathrm{h}}^{d}\right|_{\tilde{X}} \oplus i_{*} \Omega_{\mathrm{h}}^{d}\right|_{Z} \rightarrow i_{*} \pi\right|_{E *}\left(\left.\Omega_{\mathrm{h}}^{d}\right|_{E}\right)
$$

obtained from Proposition 3.3 together with the vanishing $\left.\Omega_{\mathrm{h}}^{d}\right|_{E}=0$ and $\left.\Omega_{\mathrm{h}}^{d}\right|_{Z}=0$ by Proposition 4.2 (vi) complete the proof.

Recall that any projective scheme has a dualizing sheaf in the sense of [Har77, Section III.7]. Corollary 4.7. Let $X$ be a normal projective variety of pure dimension $d$ with dualizing sheaf $\omega_{X}^{o}$. Then there exists an inclusion

$$
\left.\Omega_{\mathrm{h}}^{d}\right|_{X} \subset \omega_{X}^{o}
$$

If in addition $X$ has only rational singularities (see before Remark 6.22), then

$$
\left.\Omega_{\mathrm{h}}^{d}\right|_{X}=\omega_{X}^{o} .
$$




\section{Annette Huber and Clemens Jörder}

Proof. The dualizing sheaf is always reflexive, hence $\omega_{X}^{o}=j_{*} \Omega_{X^{\text {reg }}}^{d}$, where $j: X^{\text {reg }} \rightarrow X$ is the inclusion of the regular locus. Hence the first statement is simply Proposition 4.2(v).

Now assume that $X$ has rational singularities. Let $\pi: \tilde{X} \rightarrow X$ be a resolution. Let $\omega_{X}$ be the dualizing complex of $X$ normalized such that $\pi^{!} \omega_{X}=\Omega_{\tilde{X}}^{d}[d]$. By the definition of rational singularities, $\mathcal{O}_{X} \rightarrow R \pi_{*} \mathcal{O}_{\tilde{X}}$ is a quasi-isomorphism. By Grothendieck duality, this implies that

$$
R \pi_{*} \Omega_{\tilde{X}}^{d}[d] \rightarrow \omega_{X}
$$

is a quasi-isomorphism. Taking cohomology $H^{-d}$ in degree $-d$ yields $\omega_{X}^{0}=\pi_{*} \Omega_{\tilde{X}}^{d}$. (Note that the dualizing sheaf $\omega^{o}$ is the first non-vanishing cohomology sheaf $H^{-d} \omega_{X}$ of the dualizing complex.)

Remark 4.8. The above proof does not use the fact that a variety with rational singularities is Cohen-Macaulay. Hence it avoids the use of Kodaira vanishing.

We now turn to varieties with special types of singularities. We say that a scheme $X$ has normal crossings if $X$ is Zariski-locally isomorphic to a normal crossings divisor in a smooth variety or equivalently, if $X$ is étale locally isomorphic to a union of coordinate hyperplanes in the affine space. Observe that this implies that the irreducible components of some étale cover are smooth.

Proposition 4.9 (Normal crossing schemes). Let $X$ be a scheme with normal crossings. Then $\left.\Omega_{h}^{p}\right|_{X}=\Omega_{X}^{p} /$ torsion.

Proof. There is a natural inclusion $\Omega_{X}^{p} /$ torsion $\left.\subset \Omega_{\mathrm{h}}^{p}\right|_{X}$ by Proposition 4.2(iv). We may work étale locally in order to show that it is an isomorphism. Hence we can assume that $X$ is a union of coordinate hyperplanes in the affine space.

We prove the claim by induction on the number $c(X)$ of irreducible components of $X$, the case $c(X) \leqslant 1$ following from Proposition 4.2(ii). For $c(X)>1$ choose some irreducible component $E \subset X$ and let $X^{\prime}=\overline{X \backslash E}$. The blow-up sequence associated with $\left(X^{\prime} \rightarrow X, E \rightarrow X\right)$ fits into a commutative diagram

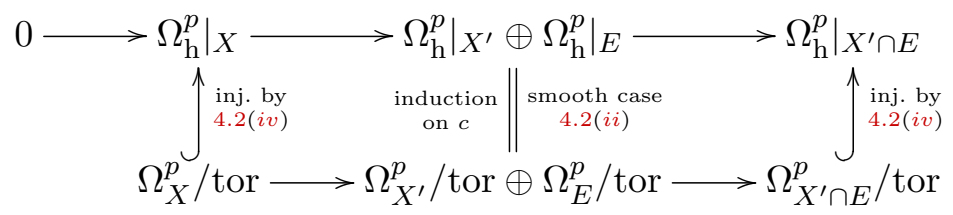

of sheaves on $X$, where the horizontal maps in the second row are given by the pull-back of torsion-free differential forms constructed in [Fer70, Proposition 1.1]. By a diagram chase one reduces the proof to showing that the second row is exact. This can be checked by a calculation using local equations for $X$ in a smooth ambient space.

Proposition 4.10 (Quotient singularities). Let $X$ be a smooth quasi-projective variety with an operation of a finite group $G$. Then $\left.\Omega_{h}^{p}\right|_{X / G}=\left(\Omega_{X}^{p}\right)^{G}=\Omega_{X / G}^{[p]}$.

Proof. Recall that $X / G$ is normal since $X$ is so and locally $X / G=\operatorname{Spec}\left(A^{G}\right)$, where $X=$ $\operatorname{Spec}(A)$. Moreover, $X \rightarrow X / G$ is a ramified cover with Galois group $G$. The first assertion is immediate from Lemma 2.10. The second was established by Knighten for $p=1$ in [Kni73] and for general $p$ by Lecomte and Wach in [LW09].

The case of klt singularities will be treated in Section 5; see Theorem 5.4. 


\section{DiFFERENTIAL FORMS IN THE h-TOPOLOGY}

\section{Application: Reflexive forms on klt base spaces}

In this section we examine the sheaf of h-differential forms on a complex variety whose singularities are mild in the sense of the Minimal Model Program. More precisely, we are concerned with the following class of singular varieties.

Definition 5.1. An irreducible variety $X$ over the complex numbers is said to be a klt base space if there exists an effective $\mathbb{Q}$-divisor $\Delta \geqslant 0$ such that the pair $(X, \Delta)$ has Kawamata $\log$ terminal singularities (see [KM98, Definition 2.34]).

Remark 5.2. In Definition 5.1 we follow the terminology in [Keb13, Definition 5.1]. This notion is equivalent to the definition of log terminal singularities in the sense of [dFH09, Theorem 1.2].

Example 5.3. A normal toric variety $X$ is locally a klt base space. Indeed, a normal projective toric variety is a klt base space by [CLS11, Example 11.4.26].

Recall that a klt base space $X$ is normal by definition, so that $\left.\Omega_{\mathrm{h}}^{p}\right|_{X} \subset \Omega_{X}^{[p]}$ by Proposition $4.2(\mathrm{v})$. The following theorem establishes the inverse inclusion and will be proved at the end of the section.

TheOrem 5.4. Let $X$ be a klt base space and $p \geqslant 0$. Then

$$
\left.\Omega_{\mathrm{h}}^{p}\right|_{X}=\Omega_{X}^{[p]} .
$$

Remark 5.5. Theorem 5.4 applies in particular to normal toric varieties by Example 5.3. In view of Corollary 2.8 this case has already been proved in [CHWW09, Theorem 4.1].

In order to deduce Theorem 5.4 from results on reflexive differential forms obtained by Greb, Kebekus, Kovács and Peternell, we need a result on the pull-back of h-differential forms under morphisms with rationally chain connected fibres proved in the next subsection, which may be of independent interest.

As an application we find a more conceptual proof of a recent result of Kebekus.

Corollary 5.6 [Keb13]. If $f: X \rightarrow Y$ is a morphism between klt base spaces, then there is a natural pull-back map

$$
\Omega^{[p]}(Y) \rightarrow \Omega^{[p]}(X)
$$

compatible with pull-back in the smooth case. That is, $\Omega^{[p]}$ is a presheaf on the category of klt base spaces.

The assumption on the type of singularities of $X$ in Theorem 5.4 seems to be optimal, since even for log canonical singularities the theorem fails.

Example 5.7 [Keb13, Section 1.2]. Let $E \subset \mathbb{P}_{\mathbb{C}}^{2}$ be an elliptic curve and let $X \subset \mathbb{A}^{3}$ be the affine cone over $E$, with vertex $p \in X$ and projection map $\pi: X \backslash\{p\} \rightarrow E$. Recall that $X$ has $\log$ canonical singularities. We claim that the inclusion

$$
\left.\Omega_{\mathrm{h}}^{1}\right|_{X} \subsetneq \Omega_{X}^{[1]}
$$

is strict. For a non-zero $\alpha \in H^{0}\left(E, \Omega_{E}^{1}\right)$ we get a non-zero element $\pi^{*}(\alpha) \in H^{0}\left(X \backslash\{p\}, \Omega^{1}\right)=$ $\Omega^{[1]}(X)$. Suppose that $\pi^{*}(\alpha)=\alpha^{\prime} \in \Omega_{\mathrm{h}}^{1}(X) \subset \Omega^{[1]}(X)$. Let $\phi: \tilde{X} \rightarrow X$ denote the blow-up of the vertex with exceptional set $\operatorname{Exc}(\phi) \cong E$. It is an $\mathbb{A}^{1}$-bundle over $E$ with $\alpha^{\prime}$ the pull-back of $\alpha$. We find a contradiction by calculating

$$
\alpha=\left.\left(\phi^{*} \alpha^{\prime}\right)\right|_{\operatorname{Exc}(\phi)}=\left.\phi\right|_{\operatorname{Exc}(\phi)} ^{*}\left(\left.\alpha^{\prime}\right|_{\{p\}}\right)=0 .
$$




\section{Annette Huber and Clemens Jörder}

\subsection{Rationally chain connected fibrations}

In this section, $k$ is an arbitrary field of characteristic 0 .

Definition 5.8. Suppose that $k$ is algebraically closed. We say that a $k$-scheme $X$ is rationally chain connected if there exists a family $g: U \rightarrow B$ of proper curves together with a morphism $U \rightarrow X$ such that

(i) the fibre $U_{b}$ over any $k$-valued point $b$ of $B$ is connected and has only rational irreducible components;

(ii) the morphism $U \times{ }_{B} U \rightarrow X \times X$ is dominant.

Lemma 5.9 [Kol96, Corollary IV.3.5.1]. Suppose that $k$ is algebraically closed. Let $X$ be a proper and rationally chain connected scheme. Then for arbitrary closed points $x_{1}, x_{2} \in X$ there exists a proper connected curve $C$ with rational irreducible components together with a morphism $C \rightarrow X$ whose image contains $x_{1}$ and $x_{2}$.

LEMma 5.10. Suppose that $k$ is algebraically closed. Let $C$ be a proper one-dimensional connected scheme all of whose irreducible components are rational curves, and let $X$ be an arbitrary scheme. Then the pull-back by the first projection $p_{X}: X \times C \rightarrow X$ yields a bijection

$$
\Omega_{\mathrm{h}}^{p}(X)=\Omega_{\mathrm{h}}^{p}(X \times C)
$$

for any $p \geqslant 0$.

Proof. It suffices to check the assertion locally in the $h$-topology. In particular, we may assume that $X$ is smooth. In the case $C=\mathbb{P}^{1}$ the assertion is an immediate consequence of Theorem 3.6 and the formula

$$
\Omega_{X \times \mathbb{P}^{1}}^{p}=\operatorname{pr}_{X}^{*}\left(\Omega_{X}^{p}\right) \oplus\left(\operatorname{pr}_{X}^{*}\left(\Omega_{X}^{p-1}\right) \otimes \operatorname{pr}_{\mathbb{P}^{1}}^{*}\left(\Omega_{\mathbb{P}^{1}}^{1}\right)\right),
$$

since the right-hand summand has no non-zero global sections.

In the general case we may assume that $C$ is reduced. Let $C^{\nu}$ be the normalization of $C$ and let $C_{\text {sing }}$ be the singular locus with its reduced scheme structure. Then $\left(C^{\nu} \rightarrow C, C_{\text {sing }} \rightarrow C\right)$ is an abstract blow-up. We denote by $E=C^{\nu} \times{ }_{C} C_{\text {sing }}$ the inverse image of $C_{\text {sing }}$ in $C^{\nu}$. The case $C=\mathbb{P}^{1}$ treated above shows that the Cartesian blow-up square given by Proposition 3.3 is

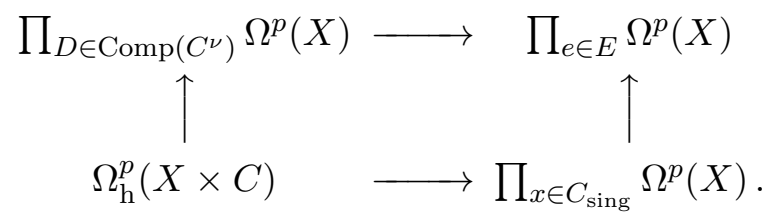

and the assertion follows using the connectedness of $C$.

Lemma 5.11. Suppose that $k$ is uncountable and algebraically closed. Let $f: X \rightarrow Y$ be a projective morphism between $k$-schemes such that

(i) the fibre $X_{y}$ over any closed point $y \in Y$ is a rationally chain connected $k$-scheme;

(ii) there exists a section $s: Y \rightarrow X$, that is, $f \circ s=\operatorname{id}_{Y}$.

Then there exist a one-dimensional proper connected scheme $C$ with rational irreducible components and a scheme $H$ together with a dominant morphism

$$
\psi: H \times Y \times C \rightarrow X
$$

over $Y$ such that for some closed point $c \in C$ the map $\psi$ satisfies $\psi(b, y, c)=s(y)$ for all closed points $b \in B, y \in Y$. 


\section{DifFERENTIAL FORMS IN THE h-TOPOLOGY}

Proof. First we introduce notation. For integers $r \geqslant 0,1 \leqslant i \leqslant r$, let $l_{i} \subset \mathbb{P}^{r}$ be the line defined by the equations $x_{j}=0$ for $0 \leqslant j \leqslant r$ and $j \neq i, i-1$. We denote by $C_{r} \subset \mathbb{P}^{r}$ the union of all $l_{i}$, $1 \leqslant i \leqslant r$ with its reduced scheme structure. Finally, let $c_{r}:=[1: 0: \cdots: 0] \in C_{r}$.

By Lemma 5.9 any two closed points of $X$ lying in the same fibre $f^{-1}(y)$ over some closed point $y \in Y$ can be connected by a proper curve $C \subset X$ with rational irreducible components, and we may even assume $C \cong C_{r}$ for some $r \geqslant 0$.

Let $H_{r}:=H_{0 m}\left(Y \times C_{r}, X\right)$ be the relative $H o m$-scheme parameterizing morphisms $Y \times$ $C_{r} \rightarrow X$ over $Y$ whose restriction to $Y \cong Y \times\left\{c_{r}\right\} \subset Y \times C_{r}$ coincides with $s$. Recall that $H_{r}$ is a countable union of schemes of finite type over $k$ by [Kol96, Theorem 1.10].

The above considerations show that the universal morphism

$$
\phi=\bigcup_{r} \phi_{r}: \bigcup_{r \geqslant 0} H_{r} \times Y \times C_{r} \rightarrow Y
$$

is surjective on $k$-points. Since the field $k$ is uncountable and the image of $\phi_{r}$ is contained in that of $\phi_{r+1}$, there exist an $r \geqslant 0$ and an open subscheme $H \subset H_{r}$ of finite type over $k$ such that the morphism $H \times Y \times C_{r} \rightarrow Y$ is dominant; see [Har77, Exercise V.4.15(c)].

TheOREm 5.12. Let $f: X \rightarrow Y$ be a surjective projective morphism between $k$-schemes such that the fibre over any $\bar{k}$-valued point of $Y$ is a rationally chain connected $\bar{k}$-scheme. Then the pull-back map $\Omega_{\mathrm{h}}^{p}(Y) \stackrel{\cong}{\rightarrow} \Omega_{\mathrm{h}}^{p}(X)$ yields a bijection for any $p \geqslant 0$.

Proof. By base change we may assume that the field $k$ is algebraically closed and uncountable.

Let us first reduce the proof to the case when $f: X \rightarrow Y$ admits a section $s: Y \rightarrow X$. To this end, we perform a base change by $f: X \rightarrow Y$. Writing $W=X \times_{Y} X$ we find a commutative diagram

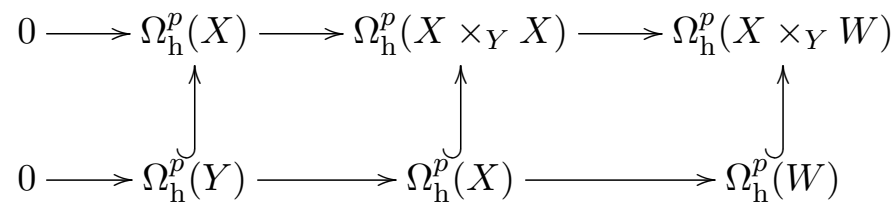

whose rows are exact and whose vertical maps are injective because they are induced by $\mathrm{h}$ covers. In particular, the theorem holds for $f: X \rightarrow Y$ if it holds for the first projection $\operatorname{pr}_{1}: X \times_{Y} X \rightarrow X$, which admits a section.

From now on we assume that $f: X \rightarrow Y$ admits a section $s: Y \rightarrow X$. Let $\psi: H \times Y \times C \rightarrow X$ and $v \in V$ be as in Lemma 5.11. In particular, we have a commutative diagram

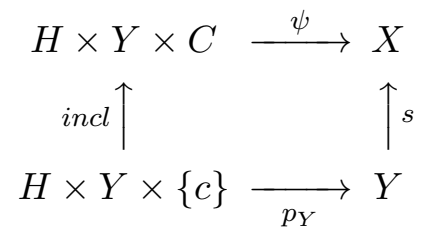

which yields a commutative diagram

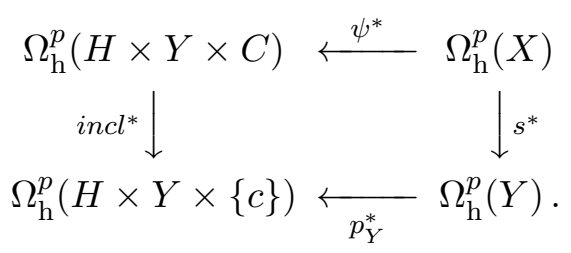




\section{Annette Huber and Clemens Jörder}

Recall that by Lemma 2.9 the pull-back $\psi^{*}$ under the dominant map $\psi$ is injective. Moreover, incl $^{*}$ is bijective by Lemma 5.10. We deduce that the pull-back $s^{*}$ under the section $s$ is injective. By

$$
\text { id }: \Omega_{\mathrm{h}}^{p}(Y) \stackrel{f^{*}}{\rightarrow} \Omega_{\mathrm{h}}^{p}(X) \stackrel{s^{*}}{\rightarrow} \Omega_{\mathrm{h}}^{p}(Y)
$$

this proves the assertion.

Remark 5.13. If $f$ and $Y$ are in addition smooth the theorem is classical. In the case $k=\mathbb{C}$, $Y=p t$ and $X$ an irreducible projective variety with Kawamata log terminal singularities, the analogous result was proved in [GKKP11, Theorem 5.1] for reflexive differentials.

\subsection{Proof of Theorem 5.4}

Let $\pi: \tilde{X} \rightarrow X$ be a resolution of singularities. The main result of [GKKP11] states that $\Omega^{[p]}(X)=\Omega^{p}(\tilde{X})$. On the other hand, [HM07, Corollary 1.5] asserts that all fibres of $\pi$ are rationally chain connected, so that $\Omega_{\mathrm{h}}^{p}(X)=\Omega_{\mathrm{h}}^{p}(\tilde{X})=\Omega^{p}(\tilde{X})$ by Theorem 5.12 .

\section{Cohomology of differential forms}

We now study the cohomology of sheaves of differential forms in the h-topology. We will first assemble some technical tools on the cohomology of h-sheaves and then apply them to the case of differential forms.

\subsection{Cohomology of h-sheaves}

Proposition 6.1. Let $\mathcal{F}$ be a sheaf of $\mathbb{Q}$-vector spaces on $S c h_{\mathrm{h}}$, let $X \in \operatorname{Sch}$ and let $p \geqslant 0$. Then

$$
H_{\mathrm{h}}^{p}(X, \mathcal{F})=H_{\mathrm{eh}}^{p}(X, \mathcal{F}) .
$$

Proof. Let $\eta: \mathrm{Sch}_{\mathrm{h}} \rightarrow \mathrm{Sch}_{\mathrm{eh}}$ be the morphism of sites. We need to show $R^{p} \eta_{*} \mathcal{F}=0$ for $p \geqslant 1$.

There exists a short exact sequence $0 \rightarrow \mathcal{F} \rightarrow \mathcal{I} \rightarrow \mathcal{G} \rightarrow 0$ of h-sheaves of $\mathbb{Q}$-vector spaces, where $\mathcal{I}$ is an injective object in the category of h-sheaves of $\mathbb{Q}$-vector spaces. It is automatically injective as sheaf of abelian groups. Since $R^{p+1} \eta_{*} \mathcal{F} \cong R^{p} \eta_{*} \mathcal{G}$ for $p \geqslant 1$, it suffices to show that $R^{1} \eta_{*} \mathcal{F}=0$ for all $\mathcal{F}$.

The sheaf $R^{1} \eta_{*} \mathcal{F}$ is the eh-sheafification of the presheaf $X \mapsto H_{\mathrm{h}}^{1}(X, \mathcal{F})$. We only need to show that $H_{\mathrm{h}}^{1}(X, \mathcal{F})=H_{\mathrm{eh}}^{1}(X, \mathcal{F})$. If $t$ is a Grothendieck topology on Sch, then by [Mil80, Corollary III.2.10],

$$
H_{t}^{1}(X, \mathcal{F})=\lim _{\mathfrak{U}} \check{H}^{1}(\mathfrak{U}, \mathcal{F})
$$

where $\mathfrak{U}$ runs through the system of $t$-covers of $X$. We apply this to $t$ equal to $\mathrm{h}$, eh, et (étale topology) and qfh (quasi-finite h; covers are h-covers which are quasi-finite). We introduce the notation $t(X)$ for the system of $t$-covers of $X$. By Proposition 3.4, any $h$-cover $\mathfrak{U}$ can be refined by a composition of a qfh-cover followed by an eh-cover $X^{\prime} \rightarrow X$ with $X^{\prime}$ smooth. Hence

$$
H_{\mathrm{h}}^{1}(X, \mathcal{F})=\lim _{\mathfrak{U} \in \mathrm{h}(X)} \check{H}^{1}(\mathfrak{U}, \mathcal{F})=\lim _{X^{\prime} \in \operatorname{eh}(X)} \lim _{\mathfrak{U} \in \mathrm{qfh}\left(X^{\prime}\right)} \check{H}^{1}(\mathfrak{U}, \mathcal{F})
$$

with $X^{\prime}$ smooth. By [Voe96, Theorem 3.4.1] we have

$$
\lim _{\mathfrak{U} \in \mathrm{qfh}\left(X^{\prime}\right)} \check{H}^{1}(\mathfrak{U}, \mathcal{F})=H_{\mathrm{qfh}}^{1}\left(X^{\prime}, \mathcal{F}\right)=H_{\mathrm{et}}^{1}\left(X^{\prime}, \mathcal{F}\right)=\lim _{\mathfrak{U} \in \operatorname{et}\left(X^{\prime}\right)} \check{H}^{1}(\mathfrak{U}, \mathcal{F}),
$$




\section{DiFFERENTIAL FORMS IN THE h-TOPOLOGY}

because $X^{\prime}$ is smooth and $\mathcal{F}$ a sheaf of $\mathbb{Q}$-vector spaces. This implies

$$
H_{\mathrm{h}}^{1}(X, \mathcal{F})=\lim _{X^{\prime} \in \operatorname{eh}(X)} \lim _{\mathfrak{U} \in \operatorname{et}\left(X^{\prime}\right)} \check{H}^{1}(\mathfrak{U}, \mathcal{F})=\lim _{\mathfrak{U} \in \operatorname{eh}(X)} \check{H}^{1}(\mathfrak{U}, \mathcal{F})=H_{\mathrm{eh}}^{1}(X, \mathcal{F}),
$$

because étale covers are eh-covers.

Proposition 6.2 (Blow-up sequence). Let $\left(X^{\prime}, Z\right)$ be an abstract blow-up of $X$ with $E=$ $X^{\prime} \times_{X} Z$. Let $\mathcal{F}$ be an h-sheaf. Then the blow-up sequence

$$
\cdots \rightarrow H_{\mathrm{h}}^{i}(X, \mathcal{F}) \rightarrow H_{\mathrm{h}}^{i}\left(X^{\prime}, \mathcal{F}\right) \oplus H_{\mathrm{h}}^{i}(Z, \mathcal{F}) \rightarrow H_{\mathrm{h}}^{i}(E, \mathcal{F}) \rightarrow H_{\mathrm{h}}^{i+1}(X, \mathcal{F}) \rightarrow \ldots
$$

is exact.

Proof. The argument is given by Geisser in [Gei06, Proposition 3.2] for the eh-topology and can be applied to the h-topology without changes.

Proposition 6.3. Let $X \in \mathrm{Sch}$ with semi-normalization $X^{\mathrm{sn}}$ (see Definition 4.3) and let $\mathcal{F}$ be an h-sheaf of abelian groups. Then

$$
H_{\mathrm{h}}^{i}(X, \mathcal{F})=H_{\mathrm{h}}^{i}\left(X^{\mathrm{sn}}, \mathcal{F}\right) .
$$

Proof. We take up the notation of the proof of Proposition 4.5, that is, let $\tilde{X}$ be the presheaf $T \mapsto X(T)$ and let $L(X)$ be its h-sheafification. By [Voe96, Theorem 3.2.9], the semi-normalisation induces an isomorphism of sheaves of sets $L\left(X^{\mathrm{sn}}\right) \cong L(X)$. This implies an isomorphism of $\mathrm{h}$-sheaves of abelian groups $\mathbb{Z}_{\mathrm{h}}\left(X^{\mathrm{sn}}\right) \rightarrow \mathbb{Z}_{\mathrm{h}}(X)$. Hence

$$
H_{\mathrm{h}}^{i}(X, \mathcal{F})=\operatorname{Ext}^{i}\left(\mathbb{Z}_{\mathrm{h}}(X), \mathcal{F}\right) \cong \operatorname{Ext}^{i}\left(\mathbb{Z}_{\mathrm{h}}\left(X^{\mathrm{sn}}\right), \mathcal{F}\right)=H_{\mathrm{h}}^{i}\left(X^{\mathrm{sn}}, \mathcal{F}\right) \text {. }
$$

\subsection{Cohomology of h-differential forms}

Corollary 6.4. Let $X \in \mathrm{Sch}$ and $X^{\mathrm{sn}}$ be the semi-normalization (see Definition 4.3) of $X$. Then

$$
H_{\mathrm{h}}^{i}\left(X, \Omega_{\mathrm{h}}^{p}\right)=H_{\mathrm{h}}^{i}\left(X^{\mathrm{sn}}, \Omega_{\mathrm{h}}^{p}\right) .
$$

Proof. This is a special case of Proposition 6.3.

Corollary 6.5 (Smooth varieties). If $X \in \mathrm{Sm}$, then

$$
H_{\mathrm{h}}^{i}\left(X, \Omega_{\mathrm{h}}^{p}\right)=H_{\mathrm{eh}}^{i}\left(X, \Omega_{\mathrm{eh}}^{p}\right)=H_{\mathrm{Zar}}^{i}\left(X, \Omega^{p}\right) .
$$

Proof. This follows from Proposition 6.1 and [Gei06, Theorem 4.7].

Remark 6.6. This was first proved with a different argument in a version for Sm equipped with the h-topology by Lee; see [Lee09, Proposition 4.2].

Corollary 6.7 (Cohomological dimension). Let $X \in \mathrm{Sch}$. Then

$$
H_{\mathrm{h}}^{i}\left(X, \Omega_{\mathrm{h}}^{p}\right)=0 \quad \text { for } i>\operatorname{dim} X .
$$

Proof. We argue by induction on the dimension of $X$, the case $\operatorname{dim}(X)<0$ being trivial. By Corollary 6.4 we may assume that $X$ is reduced, so that there exists an abstract blow-up $\left(X^{\prime}, Z\right)$ such that $X^{\prime}$ is smooth and $\operatorname{dim}(Z)<\operatorname{dim}(X)>\operatorname{dim}\left(Z \times_{X} X^{\prime}\right)$. Using the blow-up sequence 6.2, the claim for $X$ follows from that for the smooth scheme $X^{\prime}$ done by Corollary 6.5 and the inductive hypothesis applied to $Z$ and $X^{\prime} \times{ }_{X} Z$. 


\section{Annette Huber and Clemens Jörder}

Corollary 6.8 (Vanishing). Let $X$ be a variety of dimension $d$. Then

$$
H_{\mathrm{h}}^{i}\left(X, \Omega_{\mathrm{h}}^{p}\right)=0 \text { for } p>d .
$$

Proof. The argument is the same as for the cohomological dimension.

Corollary 6.9 (Finiteness). Let $X$ be proper. Then $H_{\mathrm{h}}^{i}\left(X, \Omega_{\mathrm{h}}^{p}\right)$ is finite dimensional.

Proof. The assertion holds for smooth proper $X$ because $\Omega_{X}^{p}$ is coherent. The general case follows by induction on the dimension from the blow-up sequence.

Recall the notion of an h-hypercover, for example from [Del74, Definition 5.3.4]. It is a morphism of simplicial schemes $X_{\bullet} \rightarrow Y_{\bullet}$ satisfying certain conditions which ensure that

$$
H_{\mathrm{h}}^{i}\left(Y_{\bullet}, \mathcal{F}\right) \rightarrow H_{\mathrm{h}}^{i}\left(X_{\bullet}, \mathcal{F}\right)
$$

is an isomorphism for all h-sheaves $\mathcal{F}$.

Proposition 6.10. Let $X \in \mathrm{Sch}$ and $X_{\bullet} \rightarrow X$ an h-hypercover such that all $X_{n}$ are smooth. Then

$$
H_{\mathrm{h}}^{i}\left(X, \Omega_{\mathrm{h}}^{p}\right) \cong H_{\mathrm{Zar}}^{i}\left(X_{\bullet}, \Omega_{X_{\bullet}}^{p}\right) .
$$

There is a natural spectral sequence

$$
E_{1}^{n m}=H_{\mathrm{Zar}}^{m}\left(X_{n}, \Omega_{X_{n}}^{p}\right) \Rightarrow H_{\mathrm{h}}^{n+m}\left(X, \Omega_{\mathrm{h}}^{p}\right) .
$$

Proof. This follows from Corollary 6.5 and the general descent formalism as explained for example in [Del74, Section 5.3].

The blow-up sequence easily allows the computation of cohomology with coefficients in the canonical sheaf.

Example 6.11. Let $X$ be a variety of dimension $d$. Let $X_{1}, \ldots, X_{n}$ be the irreducible components of $X$ which have dimension $d$. Let $\tilde{X}_{j} \rightarrow X_{j}$ be a resolution. Then

$$
H_{\mathrm{h}}^{i}\left(X, \Omega_{\mathrm{h}}^{d}\right)=\bigoplus_{j=1}^{n} H_{\mathrm{Zar}}^{i}\left(\tilde{X}_{j}, \Omega_{\tilde{X}_{i}}^{d}\right) .
$$

Proof. Let $X_{n+1}, \ldots, X_{N}$ be the irreducible components of $X$ whose dimension is strictly less than $d$. Let $X^{\prime}=\coprod_{j=1}^{N} X_{j}$. Then there is an abstract blow-up $\left(\pi: X^{\prime} \rightarrow X, Z\right)$ with $Z$ the locus where irreducible components intersect. Both $Z$ and its preimage in $X^{\prime}$ have dimension strictly smaller than $d$. By Corollary 6.8 its cohomology with coefficients in $\Omega_{\mathrm{h}}^{d}$ vanishes. By the blow-up sequence this implies

$$
H_{\mathrm{h}}^{i}\left(X, \Omega_{\mathrm{h}}^{d}\right)=H_{\mathrm{h}}^{i}\left(X^{\prime}, \Omega_{\mathrm{h}}^{d}\right)=\bigoplus_{j=1}^{N} H_{\mathrm{h}}^{i}\left(X_{j}, \Omega_{\mathrm{h}}^{d}\right) .
$$

The components of dimension smaller than $d$ do not contribute, by the same argument. Finally, let $Z_{j} \subset X_{j}$ be the locus where $\tilde{X}_{j} \rightarrow X_{j}$ is not an isomorphism. We use the blow-up sequence for $\left(\tilde{X}_{j} \rightarrow X_{j}, Z_{j}\right)$ to conclude.

\subsection{The derived push-forward}

After studying the h-cohomology of h-differential forms in the preceding subsection we systematically compare h-cohomology with Zariski cohomology. From now on, we work in the derived category of abelian Zariski sheaves. 


\section{DifFERENTIAL FORMS IN THE h-TOPOLOGY}

Definition 6.12. Let $\rho: \mathrm{Sch}_{\mathrm{h}} \rightarrow \mathrm{Sch}_{\mathrm{Zar}}$ be the canonical morphism of sites. For $X \in \mathrm{Sch}$ we denote by

$$
\rho_{X}: \operatorname{Sch}_{\mathrm{h}} \rightarrow X_{\mathrm{Zar}}
$$

the inclusion of $X$ with the Zariski topology. By abuse of notation, we also denote by

$$
\rho:\left(\mathrm{Sch}_{\mathrm{h}}\right)^{\sim} \rightarrow\left(\mathrm{Sch}_{\mathrm{Zar}}\right)^{\sim}, \rho_{X}:\left(\mathrm{Sch}_{\mathrm{h}}\right)^{\sim} \rightarrow X_{\text {Zar }}^{\sim}
$$

the induced morphisms of topoi.

Remark 6.13. There are also versions with the étale topology instead of the Zariski topology. For our purposes it does not make a difference which is used.

Note that $\rho^{*}$ is nothing but the h-sheafification. It is exact. The functor $\rho_{*}$ is left exact. We are going to consider its right derived functor. In accordance with Definition 4.1 we write suggestively

$$
\left.\mathcal{F}\right|_{X}=\rho_{X *} \mathcal{F} .
$$

Proposition 6.14. Let $X$ be smooth. Then the adjunction map $\Omega_{X}^{p} \rightarrow R \rho_{X *} \Omega_{\mathrm{h}}^{p}$ is a quasiisomorphism, that is,

$$
R^{i} \rho_{X *} \Omega_{\mathrm{h}}^{p}= \begin{cases}\Omega_{X}^{p}, & i=0 \\ 0, & i>0\end{cases}
$$

Proof. This is a reformulation of Corollary 6.5.

As in the absolute case this also allows us to compute $\rho_{X *}$ for singular spaces. Our main tool is again the blow-up sequence.

Proposition 6.15 (Blow-up triangle). Let $\left(\pi: X^{\prime} \rightarrow X, i: Z \rightarrow X\right)$ be an abstract blow-up of $X$ with $\pi^{\prime}: E=X^{\prime} \times_{X} Z \rightarrow Z$. Let $\mathcal{F}$ be an h-sheaf. Then there is a natural distinguished triangle

$$
R \rho_{X *} \mathcal{F} \rightarrow R \pi_{*} R \rho_{X^{\prime} *} \mathcal{F} \oplus i_{*} R \rho_{Z *} \mathcal{F} \rightarrow i_{*} R \pi_{E *}^{\prime} R \rho_{E *} \mathcal{F} \stackrel{[1]}{\longrightarrow}
$$

Proof. Like Proposition 6.2 this can be proved using the same arguments as in [Gei06, Proposition 3.2].

Corollary 6.16. Let $X \in \mathrm{Sch}$ and let $\pi: X_{\bullet} \rightarrow X$ be a proper hypercover with $X_{n}$ smooth for all $n$. Then

$$
R \rho_{X *} \Omega_{\mathrm{h}}^{p}=R \pi_{*} \Omega_{X \bullet}^{p} .
$$

Moreover, we have the following properties:

(i) The complex is concentrated in degrees at most $\operatorname{dim} X$.

(ii) It vanishes for $p>\operatorname{dim} X$.

(iii) All cohomology sheaves are coherent.

Proof. This is the sheafification of Proposition 6.10. The sheafification of Corollary 6.7 gives assertion (i). Using an induction on dimension and the blow-up triangle in Proposition 6.15, one can deduce assertion (ii) easily from Proposition 6.14. Assertion (iii) follows because $\Omega_{X}^{p}$ is coherent and $\pi$ proper. 


\section{Annette Huber And Clemens Jörder}

Remark 6.17. By construction this says that the (shifted) $p$-th graded piece $\underline{\Omega}_{X}^{p}$ of the Du Bois complex is nothing but $R \rho_{X *} \Omega_{\mathrm{h}}^{p}$. See Section 7.2 for a more detailed discussion. In particular, all statements about $R \rho_{X *} \Omega_{\mathrm{h}}^{p}$ in this section can be read as statements about $\underline{\Omega}_{X}^{p}$.

These methods also allow us to compute explicit cases.

Corollary 6.18. Let $X$ be a normal crossing scheme (see Proposition 4.9) . Then

$$
\left.\Omega_{\mathrm{h}}^{p}\right|_{X} \rightarrow R \rho_{X *} \Omega_{\mathrm{h}}^{p}
$$

is a quasi-isomorphism. In particular,

$$
H_{\mathrm{h}}^{i}\left(X, \Omega_{\mathrm{h}}^{p}\right) \cong H_{\mathrm{Zar}}^{i}\left(X,\left.\Omega_{\mathrm{h}}^{p}\right|_{X}\right)=H_{\mathrm{Zar}}^{i}\left(X, \Omega_{X}^{p} / \text { torsion }\right)
$$

Proof. The assertion can be checked étale locally. Hence we may assume that $X$ is a union of coordinate hyperplanes in the affine space.

We prove the claim by induction on the number of irreducible components of $X$, the case $X=\emptyset$ being trivial. For an arbitrary irreducible component $E \subset X$, both $X^{\prime}:=\overline{X \backslash E}$ and $E^{\prime}:=$ $E \cap X^{\prime}$ are unions of coordinate hyperplanes in affine spaces with fewer irreducible components than $X$. Write $i \bullet: \bullet \rightarrow X$ for the inclusion of a closed subscheme $\bullet$, so that the proof of Proposition 4.9 and the blow-up triangle yield a morphism of distinguished triangles

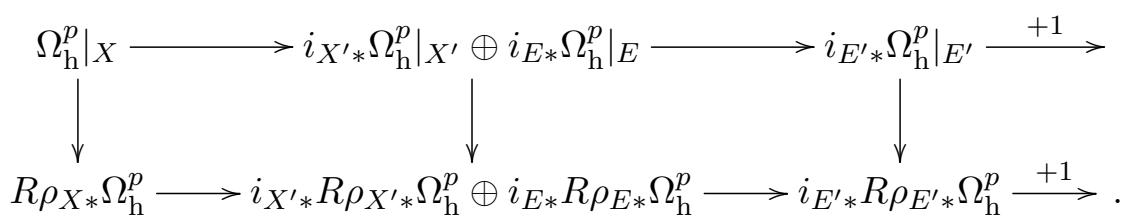

The claim follows from the induction hypothesis applied to $X^{\prime}$ and $E^{\prime}$ and Proposition 6.14 applied to $E$.

The second identification follows from Proposition 4.9.

The case of top degree is particularly interesting.

Proposition 6.19. Let $X$ be a variety of dimension $d$. Let $\pi: \tilde{X} \rightarrow X$ be a resolution, that is, proper birational with $\tilde{X}$ smooth. Then $R \rho_{X *} \Omega_{\mathrm{h}}^{d}=R \pi_{*} \Omega_{\tilde{X}}^{d}$.

Proof. The transformation $\rho_{X *} \rightarrow \pi_{*} \circ \rho_{\tilde{X} *}$ induces by Proposition 6.14 a map

$$
R \pi_{*} \Omega_{\tilde{X}}^{d}=R \pi_{*} R \rho_{\tilde{X} *} \Omega_{\mathrm{h}}^{d} \rightarrow R \rho_{X *} \Omega_{\mathrm{h}}^{d},
$$

which is a quasi-isomorphism by the sheafification of Example 6.11.

Note that this formula is independent of the choice of resolution. This allows us to give an easy proof of a statement which does not involve h-differentials at all.

Corollary 6.20. Let $\pi: \tilde{X} \rightarrow X$ be a proper birational morphism between smooth connected varieties of dimension $d$. Then $\Omega_{X}^{d} \rightarrow R \pi_{*} \Omega_{\tilde{X}}^{d}$ is a quasi-isomorphism.

Proof. We have $\Omega_{X}^{d}=R \rho_{X *} \Omega_{\mathrm{h}}^{d}=R \pi_{*} \Omega_{\tilde{X}}^{d}$ by Propositions 6.14 and 6.19.

Remark 6.21. Corollary 6.20 can be interpreted as a weak version of Grauert-Riemenschneider vanishing; see [GR70, Satz 2.3]. It is worth stressing that this vanishing results is not used in our proofs. A conceptual explanation may be that Kodaira type vanishing results are means of 


\section{DiFFERENTIAL FORMS IN THE h-TOPOLOGY}

carrying over proper base change from singular cohomology to coherent cohomology. This base change is already incorporated in our h-topology approach.

As the referee pointed out, it is tantalizing to generalize the theory to positive characteristic. For example, Corollary 6.20 would yield an alternative approach to the main results in [CR11]. However, our approach does use resolution of singularities quite heavily. In characteristic zero, it would actually be possible to use de Jong's weaker version with alterations instead. Nevertheless, a translation to positive characteristic is far from obvious because of the existence of non-separable extensions.

Recall, for example from [KM98, Definition 5.8], that a variety $X$ has rational singularities if any resolution $\pi: \tilde{X} \rightarrow X$ is rational, that is, if the adjunction map $\mathcal{O}_{X} \rightarrow R \pi_{*} \mathcal{O}_{\tilde{X}}$ is a quasi-isomorphism.

Proposition 6.22 [KM98, Theorem 5.10]. If some resolution is rational, then so is any other. In particular, if $\pi: \tilde{X} \rightarrow X$ is a proper birational map between smooth varieties, then $\mathcal{O}_{X} \rightarrow R \pi_{*} \mathcal{O}_{\tilde{X}}$ is a quasi-isomorphism.

Proof. This is a standard consequence of Corollary 6.20.

\section{Application: de Rham cohomology and the Du Bois complex}

So far, we have considered the sheaves of $p$-forms separately for every $p$. We now turn our attention to the de Rham complex and show how Du Bois singularities and the relative de Rham cohomology fit into our framework of h-sheaves.

\subsection{Cohomology of the complex of $h$-differentials}

Recall that for smooth varieties, $H_{\mathrm{Zar}}^{i}\left(X, \Omega_{X}^{*}\right)$ is called the algebraic de Rham cohomology. Following Deligne in [Del74], the definition is extended to arbitrary varieties.

Definition 7.1. Let $X \in$ Sch. Let $X \bullet \rightarrow X$ be a proper hypercover such that all $X_{n}$ are smooth. We define the algebraic de Rham cohomology of $X$ by

$$
H_{\mathrm{dR}}^{i}(X)=H_{\mathrm{Zar}}^{i}\left(X_{\bullet}, \Omega_{X \bullet}^{*}\right) .
$$

Remark 7.2. (i) By resolution of singularities such a hypercover exists.

(ii) If $k=\mathbb{C}$ and $X$ is proper, this is the de Rham component of the mixed Hodge structure on $H_{\text {sing }}^{i}\left(X^{\text {an }}, \mathbb{Q}\right)$ defined by Deligne in $\left[\right.$ Del74]. (Here $X^{\text {an }}$ denotes the analytic space attached to $X$.) In particular, $H_{\text {sing }}^{i}\left(X^{\text {an }}, \mathbb{Q}\right) \otimes_{\mathbb{Q}} \mathbb{C}$ carries a Hodge filtration $F^{p} H_{\text {sing }}^{i}\left(X^{\text {an }}, \mathbb{Q}\right) \otimes_{\mathbb{Q}} \mathbb{C}$.

(iii) For $X$ embeddable into a smooth variety, there is an alternative definition of algebraic de Rham cohomology by Hartshorne [Har75]. It is in fact equivalent; see Remark 7.5 below.

We now turn to h-topology.

DeFinition 7.3. Let $d: \Omega_{\mathrm{h}}^{p} \rightarrow \Omega_{\mathrm{h}}^{p+1}$ be the h-sheafification of the exterior differential on $p$-forms. We call

$$
\Omega_{\mathrm{h}}^{*}
$$

the algebraic de Rham complex in the h-topology. We denote by

$$
H_{\mathrm{h}}^{i}\left(X, \Omega_{\mathrm{h}}^{*}\right)
$$




\section{Annette Huber And Clemens Jörder}

its hypercohomology in the h-topology. The stupid filtration

$$
F^{p} \Omega_{\mathrm{h}}^{*}=\left[\cdots \rightarrow 0 \rightarrow \Omega_{\mathrm{h}}^{p} \rightarrow \Omega_{\mathrm{h}}^{p+1} \rightarrow \ldots\right] \subset \Omega_{\mathrm{h}}^{*}
$$

is called Hodge filtration.

Proposition 7.4 (de Rham cohomology). Let $X \in \mathrm{Sch}$. Then $H_{\mathrm{dR}}^{i}(X)=H_{\mathrm{h}}^{i}\left(X, \Omega_{\mathrm{h}}^{*}\right)$.

Proof. Let $X_{\bullet} \rightarrow X$ be a proper hypercover with all $X_{n}$ smooth. By the hypercohomology spectral sequence, that is, the spectral sequence induced by the Hodge filtration on $\Omega_{\mathrm{h}}^{*}$, and Proposition 6.10, we have

$$
H_{\mathrm{h}}^{i}\left(X, \Omega_{\mathrm{h}}^{*}\right)=H_{\mathrm{h}}^{i}\left(X_{\bullet}, \Omega_{\mathrm{h}}^{*}\right)=H_{\mathrm{Zar}}^{i}\left(X_{\bullet}, \Omega^{*}\right) .
$$

By definition, the right-hand side is the algebraic de Rham cohomology of $X$.

Remark 7.5. Geisser in [Gei06] uses the eh-topology instead. (Recall that eh-cohomology and h-cohomology agree.) He shows in loc. cit. Theorem 4.10 that the eh-cohomology of $\Omega_{\mathrm{eh}}^{*}$ agrees with Hartshorne's definition of algebraic de Rham cohomology in [Har75]. So indeed, all three approaches give the same result.

Corollary 7.6. Let $k=\mathbb{C}$. The embedding induces a natural isomorphism

$$
H_{\mathrm{h}}^{i}\left(X, \Omega_{\mathrm{h}}^{*}\right) \cong H_{\text {sing }}^{i}\left(X_{\mathbb{C}}^{\text {an }}, \mathbb{C}\right) .
$$

Proof. This is the period isomorphism between algebraic de Rham cohomology and singular cohomology.

Theorem 7.7 (Hodge filtration). Let $X \in$ Sch be proper. Then the Hodge to de Rham spectral sequence

$$
E_{1}^{a b}=H_{\mathrm{h}}^{b}\left(X, \Omega_{\mathrm{h}}^{a}\right) \Rightarrow H_{\mathrm{h}}^{a+b}\left(X, \Omega_{\mathrm{h}}^{*}\right)
$$

degenerates at $E_{1}$. Under the identification in Proposition 7.4, the Hodge filtration $F^{*} \Omega_{\mathrm{h}}^{*}$ induces the Hodge filtration on $H_{\mathrm{dR}}^{i}(X)$.

Proof. Choose a proper hypercover $X \bullet$ of $X$ with all $X_{n}$ smooth. The comparison argument in the proof of Proposition 7.4 also works for all $F^{p} \Omega_{\mathrm{h}}^{*}$, so we can replace $H_{\mathrm{h}}^{i}(X, \cdot)$ by $H_{\mathrm{Zar}}^{i}\left(X_{\bullet}, \cdot\right)$ everywhere in the assertion. Hence this is really a statement about the Zariski cohomology of differential forms.

By the base change properties of differential forms and the cohomology of coherent sheaves, the theorem is true for the ground field $k$ if and only if it is true for a field extension of $k$. The scheme $X$ and every $X_{n}$ are defined over a finitely generated extension of $\mathbb{Q}$. Hence we can assume without loss of generality that $k$ is generated over $\mathbb{Q}$ by countably many elements. Such fields can be embedded into $\mathbb{C}$, hence it suffices to consider the case $k=\mathbb{C}$.

In this case, the assertion is proved by Deligne in [Del74]: by definition

$$
F^{p} H_{\text {sing }}^{i}\left(X^{\text {an }}, \mathbb{C}\right)=H_{\text {Zar }}^{i}\left(X_{\bullet}, F^{p} \Omega^{*}\right)
$$

and the spectral sequence attached to the Hodge filtration degenerates at $E_{1}$.

Corollary 7.8. Let $k=\mathbb{C}$ and let $X$ be proper. Then the natural map

$$
H_{\text {sing }}^{i}\left(X^{\mathrm{an}}, \mathbb{C}\right) \rightarrow H_{\mathrm{h}}^{i}\left(X, \Omega_{\mathrm{h}}^{0}\right)
$$

is surjective. 


\section{DiFFERENTIAL FORMS IN THE h-TOPOLOGY}

Proof. In this case we can identify algebraic de Rham cohomology with singular cohomology.

Remark 7.9. This generalizes the well-known consequence of the degeneration of the Hodge to de Rham spectral sequence to the singular case. Note that this is the starting point in the proof of well-known vanishing theorems in birational geometry, as pointed out for example in [Kol87, Part III] and [CKM88, Lecture 8].

\subsection{The Du Bois complex}

In this subsection we point out how the Du Bois complex fits into the framework of h-differential forms. The connection was first observed by Lee in [Lee09]. In order to have a self-contained presentation, we explain the details in our language. We use the new language to reprove a number of results from the literature with very short and simple proofs.

Recall from [Del74] the notion of a filtered derived category $D^{+} F \mathcal{A}$ of an abelian category $\mathcal{A}$. Objects are filtered complexes and morphisms are morphisms of filtered complexes localized with respect to filtered quasi-isomorphisms. We assume that all filtrations are decreasing.

Du Bois defines in [DuB81] a filtered complex of sheaves on $X$ whose hypercohomology is de Rham cohomology with the Hodge filtration. We review the construction.

Definition 7.10. Let $X$ be a variety, and let $\pi: X_{\bullet} \rightarrow X$ be a proper hypercover with all $X_{n}$ smooth. Then

$$
\underline{\Omega}_{X}^{*}:=R \pi_{*} \Omega_{X}^{*}
$$

is the Du Bois complex of $X$. It is filtered by $F^{p} \underline{\Omega}_{X}^{*}=R \pi_{*} F^{p} \Omega_{X \bullet}^{*}$. We denote by

$$
\underline{\Omega}_{X}^{p}=F^{p} \underline{\Omega}_{X}^{*} / F^{p+1} \underline{\Omega}_{X}^{*}[p]=R \pi_{*} \Omega_{X}^{p}
$$

its associated graded piece.

Remark 7.11. Du Bois assumes that $X$ is proper. This is needed if we want the induced filtration on hypercohomology to be the correct Hodge filtration.

Theorem 7.12. Consider $\Omega_{\mathrm{h}}^{*}$ with the Hodge filtration as an object in $D^{+} F\left(\operatorname{Sh}\left(\mathrm{Sch}_{\mathrm{h}}\right)\right)$ where $\mathrm{Sh}\left(\mathrm{Sch}_{\mathrm{h}}\right)$ is the category of sheaves of abelian groups on $\mathrm{Sch}_{\mathrm{h}}$. Then

$$
R \rho_{X *} \Omega_{\mathrm{h}}^{*} \in D^{+} F\left(\operatorname{Sh}\left(X_{\text {Zar }}\right)\right)
$$

is naturally isomorphic to the Du Bois complex $\underline{\Omega}_{X}^{*}$. In particular,

$$
R \rho_{X *} \Omega_{\mathrm{h}}^{p} \cong \underline{\Omega}_{X}^{p} \in D\left(\operatorname{Sh}(X)_{\mathrm{Zar}}\right) .
$$

Remark 7.13. A variant of this result was proved by Lee; see [Lee09, Theorem 4.13].

Proof. Choose a proper hypercover $\pi: X \bullet \rightarrow X$ as in the definition of $\underline{\Omega}_{X}^{*}$. We need to show that $R \rho_{X *} \Omega_{\mathrm{h}}^{*} \cong R \pi_{*} \Omega_{X}^{*}$. Using the Hodge filtration on both sides, this follows from Corollary 6.16.

Corollary 7.14 [DuB81]. As an object of the filtered derived category, $\underline{\Omega}_{X}^{*}$ is independent of the choice of hypercover $\pi: X_{\bullet} \rightarrow X$.

Proof. This is true for $R \rho_{X *} \Omega_{\mathrm{h}}^{*}$.

Remark 7.15. After this identification, the statements in Section 6.3 can be understood as statements on the graded pieces of the Du Bois complex.

Corollary 7.16 [GNPP88, Proposition III.1.17]. The Du Bois complex of $X$ is a complex of coherent sheaves with $k$-linear coboundary maps concentrated in degrees at most $2 \operatorname{dim} X$. 


\section{Annette Huber And Clemens Jörder}

Proof. Use Proposition 6.16 and the Hodge spectral sequence.

Recall that in [Ste83, (3.5)] Steenbrink defined a variety to have Du Bois singularities if the canonical map from $\mathcal{O}_{X}$ to the zeroth graded piece of the Du Bois complex is a quasi-isomorphism. These singularities have already been studied by Du Bois in [DuB81, Section 4].

Corollary 7.17. A variety $X$ has Du Bois singularities if and only if $\mathcal{O}_{X} \rightarrow R \rho_{X *} \Omega_{\mathrm{h}}^{0}$ is a quasi-isomorphism, that is, if

$$
R^{i} \rho_{X *} \Omega_{\mathrm{h}}^{0}= \begin{cases}\mathcal{O}_{X} & i=0 \\ 0 & i>0 .\end{cases}
$$

In particular, if $X$ has Du Bois singularities, then $H_{\mathrm{h}}^{i}\left(X, \Omega_{\mathrm{h}}^{0}\right)=H_{\mathrm{Zar}}^{i}\left(X, \mathcal{O}_{X}\right)$.

Remark 7.18. By Proposition 4.5, we have $\rho_{X *} \Omega_{\mathrm{h}}^{0}=\mathcal{O}_{X}$ if $X$ is semi-normal. More generally, the argument shows that the zeroth cohomology group of $\underline{\Omega}_{X}^{0}$ is isomorphic to $\pi_{*} \mathcal{O}_{X^{\text {sn }}}$, where $\pi: X^{\text {sn }} \rightarrow X$ is the semi-normalization. This reproves a result established by Saito [Sai00, Proposition 5.2] or Schwede [Schw09, Lemma 5.6].

Example 7.19 [DuB81, Exemples 4.7]. Normal crossing schemes are Du Bois by Corollary 6.18 for $p=0$.

Using our approach we can also explain Schwede's criterion.

Corollary 7.20 [Schw07]. Let $X \subset Y$ be a scheme embedded in some smooth scheme $Y$ and let $\pi: \tilde{Y} \rightarrow Y$ be a $\log$ resolution of $X$ in $Y$, that is, the exceptional set $E=\operatorname{Exc}(\pi)_{\text {red }}=\pi^{-1}(X)_{\text {red }}$ is a simple normal crossing divisor. Then $X$ has Du Bois singularities if and only if the canonical $\operatorname{map} \mathcal{O}_{X} \rightarrow R \pi_{*} \mathcal{O}_{E}$ is an isomorphism.

Proof. By Proposition 6.14, Corollary 6.18 and the equality $R \pi_{*} \mathcal{O}_{\tilde{Y}}=\mathcal{O}_{Y}$ (see Remark 6.22) the blow-up triangle 6.15 for $\mathcal{O}_{\mathrm{h}}$ and $(\tilde{Y} \rightarrow Y, X \stackrel{i}{\rightarrow} Y)$ can be written as

$$
\mathcal{O}_{Y} \rightarrow \mathcal{O}_{Y} \oplus i_{*} R \rho_{X *} \mathcal{O}_{\mathrm{h}} \rightarrow R \pi_{*} \mathcal{O}_{E} \stackrel{+1}{\longrightarrow}
$$

which shows the claim.

\subsection{Relative de Rham cohomology}

Let $X$ be a variety and let $Z \rightarrow X$ be a closed subvariety. Our aim is to describe the de Rham cohomology of $X$ relative to $Z$ by h-differentials.

As mentioned in the introduction, relative de Rham cohomology is needed in a generalization of the definition of the period isomorphism and the period numbers of a variety, for example in the work of Kontsevich and Zagier on periods [KZ02]; see also [HM11]. We are not aware of a reference in the literature even though the existence of such a theory is clear to the experts.

Definition 7.21. Let $X$ be in Sch. Let $(\mathrm{Sch} / X)_{\mathrm{h}}$ be the category of schemes of finite type over $X$ equipped with the restriction of the h-topology.

Let $f: X \rightarrow Y$ be in Sch. We denote by

$$
f^{*}:(\mathrm{Sch} / Y)_{\mathrm{h}}^{\sim} \rightarrow(\mathrm{Sch} / X)_{\mathrm{h}}^{\sim}
$$

the restriction functor and by

$$
f_{*}:(\mathrm{Sch} / X)_{\mathrm{h}}^{\sim} \rightarrow(\mathrm{Sch} / Y)_{\mathrm{h}}^{\sim}
$$

its right adjoint. 


\section{DifFERENTIAL FORMS IN THE h-TOPOLOGY}

We will frequently use the following fact.

Lemma 7.22. Let $\pi: X \rightarrow$ Spec $k$ be the structural map. For any sheaf $\mathcal{F}$ of abelian groups on $\operatorname{Sch}_{\mathrm{h}}=(\operatorname{Sch} / \operatorname{Spec} k)_{\mathrm{h}}$, we have $H_{\mathrm{h}}^{i}(X, \mathcal{F})=H^{i}\left((\operatorname{Sch} / X)_{\mathrm{h}}, \pi^{*} \mathcal{F}\right)$.

Proof. This is [Mil80, Lemma III.1.11].

Definition 7.23. We denote by $\Omega_{\mathrm{h} / X}^{p}$ the restriction of $\Omega_{\mathrm{h}}^{p}$ to $(\mathrm{Sch} / X)_{\mathrm{h}}$. Equivalently, $\Omega_{\mathrm{h} / X}^{p}$ is the h-sheafification of the presheaf $\Omega^{p}$ on $\mathrm{Sch} / X$.

Definition 7.24. Let $X \in \mathrm{Sch}$ and let $i: Z \rightarrow X$ be a closed subscheme. Put

$$
\Omega_{\mathrm{h} /(X, Z)}^{p}=\operatorname{Ker}\left(\Omega_{\mathrm{h} / X}^{p} \rightarrow i_{*} \Omega_{\mathrm{h} / Z}^{p}\right)
$$

in the category of abelian sheaves on $(\mathrm{Sch} / X)_{\mathrm{h}}$.

We define the relative algebraic de Rham cohomology as

$$
H_{\mathrm{dR}}^{p}(X, Z)=H_{\mathrm{h}}^{p}\left(X, \Omega_{\mathrm{h} /(X, Z)}^{*}\right) .
$$

Remark 7.25. Relative algebraic de Rham cohomology could alternatively be defined as part of the Hodge structure on relative singular cohomology or as the de Rham realization of appropriate geometric motives (see [Hub00] and [Hub04], or [LW09]). The above agrees with these definitions. We refrain from giving the details of the comparison.

Establishing standard properties of relative algebraic de Rham cohomology in terms of hyperresolutions is very difficult. Indeed, the standard argument would be to compare the situation with singular cohomology, where the proofs are straightforward. Our approach via the h-topology allows us to give these proofs directly.

Lemma 7.26. Let $i: Z \rightarrow X$ be a closed immersion.

(i) Then $R i_{*} \Omega_{\mathrm{h} / Z}^{p}=i_{*} \Omega_{\mathrm{h} / Z}^{p}$ and hence

$$
H_{\mathrm{h}}^{q}\left(X, i_{*} \Omega_{\mathrm{h} / Z}^{p}\right)=H_{\mathrm{h}}^{q}\left(Z, \Omega_{\mathrm{h}}^{p}\right) .
$$

(ii) The natural map of sheaves of abelian groups on $(\mathrm{Sch} / X)_{\mathrm{h}}$

$$
\Omega_{\mathrm{h} / X}^{p} \rightarrow i_{*} \Omega_{\mathrm{h} / Z}^{p}
$$

is surjective.

Proof. The higher direct image $R^{q} i_{*} \Omega_{\mathrm{h} / Z}^{p}$ is the h-sheafification in $(\mathrm{Sch} / X)_{\mathrm{h}}$ of

$$
(f: U \rightarrow X) \mapsto H_{\mathrm{h}}^{q}\left(f^{-1} Z, \Omega_{\mathrm{h}}^{p}\right)
$$

We write $g: U^{\prime} \rightarrow U \rightarrow X$ where $U^{\prime} \rightarrow U$ is a resolution such that locally on $U^{\prime}$ the preimage $g^{-1} Z \subset U^{\prime}$ either is a simple normal crossing divisor or equals $U^{\prime}$. In a second step, we cover $U^{\prime}$ by open affines. Hence it suffices to show that

$$
H_{\mathrm{h}}^{q}\left(Z^{\prime}, \Omega_{\mathrm{h}}^{p}\right)=0 \quad q>0
$$

for $Z^{\prime}$ an affine scheme with normal crossings. By Corollary 6.18 it is equal to the Zariski cohomology of a coherent sheaf, hence zero.

For surjectivity, it suffices by the same reduction to show that

$$
\Omega^{p}\left(U^{\prime}\right) \rightarrow \Omega_{\mathrm{h}}^{p}\left(Z^{\prime}\right)
$$

is surjective for $U^{\prime}$ smooth affine and $Z^{\prime} \subset U^{\prime}$ a divisor with normal crossings. Note that this is true for Kähler differentials. By Proposition 4.9 , the sheaf $\left.\Omega_{\mathrm{h}}^{p}\right|_{Z^{\prime}}$ is a quotient of $\Omega_{Z}^{p}$ in the Zariski topology. 


\section{Annette Huber And Clemens Jörder}

Proposition 7.27 (Long exact sequence). Let $Z \subset Y \subset X$ be a closed immersion. Then there is a natural long exact sequence

$$
\cdots \rightarrow H_{\mathrm{dR}}^{q}(X, Y) \rightarrow H_{\mathrm{dR}}^{q}(X, Z) \rightarrow H_{\mathrm{dR}}^{q}(Y, Z) \rightarrow H_{\mathrm{dR}}^{q+1}(X, Y) \rightarrow \cdots
$$

Proof. We compute in the category of abelian sheaves on $(\mathrm{Sch} / X)_{\mathrm{h}}$. Let $i_{Y}: Y \rightarrow X, i_{Z}: Z \rightarrow X$. By definition, there is a commutative diagram

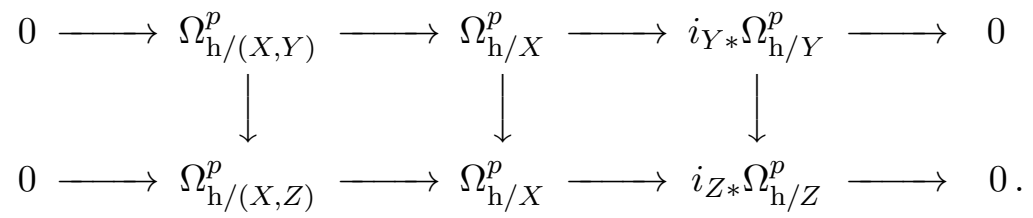

The functor $i_{Y *}$ is left exact, hence we have

$$
i_{Y *} \Omega_{\mathrm{h} /(Y, Z)}^{p}=\operatorname{Ker}\left(i_{Y *} \Omega_{\mathrm{h} / Y}^{p} \rightarrow i_{Z *} \Omega_{\mathrm{h} / Z}^{p}\right) .
$$

By the snake lemma this implies that we have a short exact sequence

$$
0 \rightarrow \Omega_{\mathrm{h} /(X, Y)}^{p} \rightarrow \Omega_{\mathrm{h} /(X, Z)}^{p} \rightarrow i_{Y *} \Omega_{\mathrm{h} /(Y, Z)} \rightarrow 0 .
$$

The long exact sequence for relative cohomology is the long exact cohomology sequence attached to it, provided we establish

Using Lemma 7.26 we obtain a natural triangle

$$
R i_{Y *} \Omega_{\mathrm{h} /(Y, Z)}^{p}=i_{Y *} \Omega_{\mathrm{h} /(Y, Z)}^{p} .
$$

$$
R i_{Y *} \Omega_{\mathrm{h} /(Y, Z)}^{p} \rightarrow i_{Y *} \Omega_{\mathrm{h} / Y}^{p} \rightarrow i_{Z *} \Omega_{\mathrm{h} / Z}^{p}
$$

By considering the long exact cohomology sequence on $(\mathrm{Sch} / X)_{\mathrm{h}}$, it suffices to show the surjectivity of

$$
i_{Y *} \Omega_{\mathrm{h} / Y}^{p} \rightarrow i_{Z *} \Omega_{\mathrm{h} / Z}^{p} .
$$

This is true because $\Omega_{\mathrm{h} / X}^{p}$ surjects to both by Lemma 7.26.

Proposition 7.28 (Excision). Let $\tilde{X} \rightarrow X$ be a proper morphism, which is an isomorphism outside of $Z \subset X$. Let $\tilde{Z}=\pi^{-1} Z$. Then $H_{\mathrm{dR}}^{q}(\tilde{X}, \tilde{Z}) \cong H_{\mathrm{dR}}^{q}(X, Z)$.

Proof. This follows immediately from the blow-up triangle.

Proposition 7.29 (Künneth formula). Let $Z \subset X$ and $Z^{\prime} \subset X^{\prime}$ be closed immersions. Then there is a natural isomorphism

$$
H_{\mathrm{dR}}^{n}\left(X \times X^{\prime}, X \times Z^{\prime} \cup Z \times X^{\prime}\right)=\bigoplus_{a+b=n} H_{\mathrm{dR}}^{a}(X, Z) \otimes_{k} H_{\mathrm{dR}}^{b}\left(X^{\prime}, Z^{\prime}\right) .
$$

Proof. We work in the category of h-sheaves of $k$-vector spaces on $X \times X^{\prime}$. Note that the hcohomology of an h-sheaf of $k$-vector spaces computed in the category of sheaves of abelian groups agrees with its h-cohomology computed in the category of sheaves of $k$-vector spaces because an injective sheaf of $k$-vector spaces is also injective as a sheaf of abelian groups.

We abbreviate $T=X \times Z^{\prime} \cup Z \times X^{\prime}$. By the h-sheafification of the product of Kähler differentials we have a natural multiplication

$$
\operatorname{pr}_{X}^{*} \Omega_{\mathrm{h} / X}^{a} \otimes_{k} \operatorname{pr}_{X^{\prime}}^{*} \Omega_{\mathrm{h} / X^{\prime}}^{b} \rightarrow \Omega_{\mathrm{h} / X \times X^{\prime}}^{a+b} .
$$

It induces, with $i_{Z}: Z \rightarrow X, i_{Z^{\prime}}: Z^{\prime} \rightarrow X^{\prime}$ and $i: T \rightarrow X \times X^{\prime}$,

$$
\operatorname{pr}_{X}^{*} \operatorname{Ker}\left(\Omega_{\mathrm{h} / X}^{a} \rightarrow i_{Z *} \Omega_{\mathrm{h} / Z}^{a}\right) \otimes_{k} \operatorname{pr}_{X^{\prime}}^{*} \operatorname{Ker}\left(\Omega_{\mathrm{h} / X^{\prime}}^{b} \rightarrow i_{Z^{\prime} *} \Omega_{\mathrm{h} / Z^{\prime}}^{b}\right) \rightarrow \operatorname{Ker}\left(\Omega_{\mathrm{h} / X \times X^{\prime}}^{a+b} \rightarrow i_{*} \Omega_{\mathrm{h} / T}^{a+b}\right) .
$$


The resulting morphism

$$
\operatorname{pr}_{X}^{*} \Omega_{\mathrm{h} /(X, Z)}^{*} \otimes_{k} \operatorname{pr}_{X^{\prime}}^{*} \Omega_{\mathrm{h} /\left(X^{\prime}, Z^{\prime}\right)}^{*} \rightarrow \Omega_{\mathrm{h} /\left(X \times X^{\prime}, T\right)}^{*}
$$

induces a natural Künneth morphism

$$
\bigoplus_{a+b=n} H_{\mathrm{dR}}^{a}(X, Z) \otimes_{k} H_{\mathrm{dR}}^{b}\left(X^{\prime}, Z^{\prime}\right) \rightarrow H_{\mathrm{dR}}^{n}\left(X \times X^{\prime}, T\right)
$$

It remains to show that it is an isomorphism. One can easily show that the Künneth morphism is compatible with long exact sequences of pairs of spaces in both arguments, possibly up to sign. For example, for the second argument the diagrams

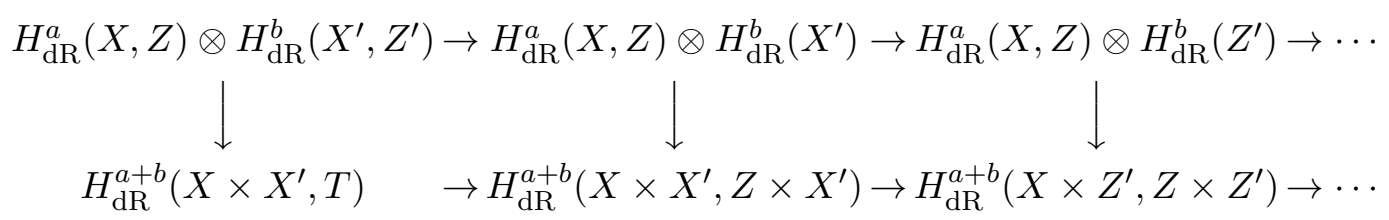

commute (possibly up to sign), where the second row is the long exact sequence associated with $Z \times X^{\prime} \subset T \subset X \times X^{\prime}$ up to the excision isomorphism

$$
H_{\mathrm{dR}}^{a+b}\left(T, X \times Z^{\prime}\right)=H_{\mathrm{dR}}^{a+b}\left(X \times Z^{\prime}, Z \times Z^{\prime}\right) .
$$

By this and a similar consideration for the first argument, we reduce to the case $Z^{\prime}=Z=\emptyset$.

In the second step, we use that the Künneth morphism is compatible with long exact sequences for abstract blow-ups, again in both arguments. Hence it suffices to show the isomorphism

$$
\bigoplus_{a+b=n} H_{\mathrm{dR}}^{a}(X) \otimes_{k} H_{\mathrm{dR}}^{b}\left(X^{\prime}\right) \rightarrow H_{\mathrm{dR}}^{n}\left(X \times X^{\prime}\right)
$$

for $X$ and $X^{\prime}$ smooth. In this case we can compute in the Zariski topology. The isomorphism is well known. It follows from the Künneth formula for the Zariski cohomology of vector bundles.

A special case of relative cohomology is cohomology with compact support.

Definition 7.30. Let $X \in$ Sch and let $j: X \rightarrow \bar{X}$ be a compactification. Then

$$
H_{\mathrm{dR}, c}^{q}(X)=H_{\mathrm{dR}}^{q}(\bar{X}, X)
$$

is called the algebraic de Rham cohomology with compact support.

In the setting of the eh-topology this is precisely [Gei06, Section 4.1]. By excision, the definition is independent of the choice of compactification.

\section{ACKNowledgements}

We are particularly thankful to Stefan Kebekus who suggested that we should work together on this project. He was the one who asked about the relation to the Du Bois complex. We are very grateful to Matthias Wendt for his careful reading of a first version of our article. We also thank them and our other colleagues at Freiburg University for comments and explanations: Daniel Greb, Patrick Graf, and Wolfgang Soergel.

\section{REFERENCES}

Bei12 A. Beilinson, p-adic periods and derived de Rham cohomology, J. Amer. Math. Soc. 25 (2012), 715-738 http://dx.doi.org/10.1090/S0894-0347-2012-00729-2 


\section{Annette Huber and Clemens Jörder}

CHSW08 G. Cortiñas, C. Haesemeyer, M. Schlichting and C. Weibel, Cyclic homology, cdh-cohomology and negative K-theory, Annals of Math. 167 (2008), no. 2, 549-573. http://dx.doi.org/10. 4007/annals. 2008.167.549

CHWW09 G. Cortiñas, C. Haesemeyer, M. Walker and C. Weibel, The K-theory of toric varieties, Trans. Amer. Math. Soc. 361 (2009), no. 6, 3325-3341. http://dx.doi.org/10.1090/ S0002-9947-08-04750-8

CHWW10 G. Cortiñas, C. Haesemeyer, M. Walker and C. Weibel, Bass' NK groups and cdh-fibrant Hochschild homology, Invent. Math. 181 (2010), no. 2, 421-448. http://dx.doi.org/10. 1007/s00222-010-0253-z

CHWW11 G. Cortiñas, C. Haesemeyer, M. Walker and C. Weibel, A negative answer to a question of Bass, Proc. Amer. Math. Soc. 139 (2011), no. 4, 1187-1200. http://dx.doi.org/10.1090/ S0002-9939-2010-10728-1

CHWW13 G. Cortiñas, C. Haesemeyer, M. Walker and C. Weibel, K-theory of cones of smooth varieties, J. Algebraic Geom. 22 (2013), no. 1, 13-34. http://dx.doi.org/10.1090/ S1056-3911-2011-00583-3

CHW08 G. Cortiñas, C. Haesemeyer and C. Weibel, K-regularity, cdh-fibrant Hochschild homology, and a conjecture of Vorst, J. Amer. Math. Soc. 21 (2008), no. 2, 547-561. http://dx.doi. org/10.1090/S0894-0347-07-00571-1

CKM88 H. Clemens, J. Kollár and S. Mori, Higher-dimensional complex geometry, Astérisque, No. 166 (1988).

CLS11 D. Cox, J. Little and H. Schenck, Toric varieties, Graduate Studies in Mathematics 124, American Mathematical Society, Providence, RI, 2011.

CR11 A. Chatzistamatiou and K. Rülling, Higher direct images of the structure sheaf in positive characteristic, Algebra Number Theory 5 (2011), no. 6, 693-775. http://dx.doi.org/10. 2140/ant.2011.5.693

Del74 P. Deligne, Théorie de Hodge. III, Inst. Hautes Etudes Sci. Publ. Math. 44 (1974), 5-77.

DuB81 P. Du Bois, Complexe de de Rham filtré d'une variété singuliére, Bull. Soc. Math. France 109 (1981), no. 1, 41-81.

Fer70 A. Ferrari, Cohomology and holomorphic differential forms on complex analytic spaces, Ann. Scuola Norm. Sup. Pisa (3) 24 (1970), 65-77.

dFH09 T. de Fernex, C. Hacon, Singularities on normal varieties, Compositio Math. 145 (2009), no. 2, 393-414. http://dx.doi.org/10.1112/S0010437X09003996

Gei06 T. Geisser, Arithmetic cohomology over finite fields and special values of $\zeta$-functions, Duke Math. J. 133 (2006), no. 1, 27-57. http://dx.doi.org/10.1215/S0012-7094-06-13312-4

GKKP11 D. Greb, S. Kebekus, S. Kovács and T. Peternell, Differential forms on log canonical spaces, Publ. Math. Inst. Hautes Etudes Sci. 114 (2011), 87-169. http://dx.doi.org/10.1007/ s10240-011-0036-0

GNPP88 F. Guillén, V. Navarro Aznar, P. Pascual Gainza and F. Puerta, Hyperrésolutions cubiques et descente cohomologique. Papers from the Seminar on Hodge-Deligne Theory held in Barcelona, 1982, Lecture Notes in Mathematics, vol. 1335, Springer-Verlag, Berlin, 1988.

GR70 H. Grauert and O. Riemenschneider, Verschwindungssätze für analytische Kohomologiegruppen auf komplexen Räumen, Invent. Math. 11 (1970), 263-292.

Gro85 M. Gros, Classes de Chern et classes de cycles en cohomologie de Hodge-Witt logarithmique, Mém. Soc. Math. France (N.S.) 21 (1985).

Har75 R. Hartshorne, On the De Rham cohomology of algebraic varieties, Inst. Hautes Etudes Sci. Publ. Math. 45 (1975), 5-99.

Har77 R. Hartshorne, Algebraic geometry, Graduate Texts in Mathematics, Vol. 52. Springer-Verlag, New York-Heidelberg, 1977. 


\section{DiFFERENTIAL FORMS IN THE h-TOPOLOGY}

HM07 C.D. Hacon and J. McKernan, On Shokurov's rational connectedness conjecture, Duke Math. J. 138 (2007), no. 1, 119-136. http://dx.doi.org/10.1215/S0012-7094-07-13813-4

HM11 A. Huber and S. Müller-Stach, On the relation between Nori Motives and Kontsevich Periods, arXiv:1105.0865 (2011).

Hub00 A. Huber, Realization of Voevodsky's motives, J. Algebraic Geom. 9 (2000), no. 4, 755-799.

Hub04 A. Huber, Corrigendum to: "Realization of Voevodsky's motives", [J. Algebraic Geom. 9 (2000), no. 4, 755-799], J. Algebraic Geom. 13 (2004), no. 1, 195-207. http://dx.doi.org/ 10.1090/S1056-3911-03-00374-6

Jör14 C. Jörder, On the Poincaré Lemma for reflexive differential forms, arXiv:1401.7495 (2014).

Keb13 S. Kebekus, Pull-back morphisms for reflexive differential forms, Adv. Math. 245 (2013), 78-112. http://dx.doi.org/10.1016/j.aim.2013.06.013

KM98 J. Kollár and S. Mori, Birational geometry of algebraic varieties, Cambridge Tracts in Mathematics, 134, Cambridge University Press, Cambridge, 1998.

Kni73 C.M. Knighten, Differentials on quotients of algebraic varieties, Trans. Amer. Math. Soc. $\mathbf{1 7 7}$ (1973), 65-89.

Kol87 J. Kollár, Vanishing theorems for cohomology groups, in Algebraic geometry, Bowdoin, 1985 (Brunswick, Maine, 1985), 233-243, Proc. Sympos. Pure Math. 46, Part 1, Amer. Math. Soc. Providence, RI, 1987.

Kol96 J. Kollár, Rational curves on algebraic varieties, Ergebnisse der Mathematik und ihrer Grenzgebiete, 3. Folge, A Series of Modern Surveys in Mathematics vol. 32, Springer-Verlag, Berlin, 1996.

Kov99 S. Kovács, Rational, log canonical, Du Bois singularities: on the conjectures of Kollár and Steenbrink, Compositio Math. 118 (1999), no. 2, 123-133.

KZ02 M. Kontsevich and D. Zagier, Periods, in Mathematics unlimited-2001 and beyond, 771-808, Springer, Berlin, 2001.

Lee09 B. Lee, Local acyclic fibrations and the de Rham complex, Homology, Homotopy Appl. 11 (2009), no. 1, 115-140. http://dx.doi.org/10.4310/HHA.2009.v11.n1.a6

LW09 F. Lecomte and N. Wach, Le complexe motivique de De Rham, Manuscripta Math. 129 (2009), no. 1, 75-90. http://dx.doi.org/10.1007/s00229-008-0248-x

Mil80 J.S. Milne, Etale cohomology Princeton Mathematical Series, 33, Princeton University Press, Princeton, N.J., 1980.

Sai00 M. Saito, Mixed Hodge complexes on algebraic varieties, Math. Ann. 316 (2000), no. 2, 283331. http://dx.doi.org/10.1007/s002080050014

Scho12 J. Scholbach, Geometric motives and the h-topology, Math. Z. 272 (2012), 965-986. http: //dx.doi.org/10.1007/s00209-011-0968-6

Schw07 K. Schwede, A simple characterization of Du Bois singularities, Compositio Math. 143 (2007), no. $4,813-828$.

Schw09 K. Schwede, F-injective singularities are Du Bois, Amer. J. Math. 131 (2009), no. 2, 445-473.

SvS85 J. Steenbrink and D. van Straten, Extendability of holomorphic differential forms near isolated hypersurface singularities, Abh. Math. Sem. Univ. Hamburg 55 (1985), 97-110.

Ste83 J.H.M. Steenbrink, Mixed Hodge structures associated with isolated singularities, in Singularities, Part 2 (Arcata, Calif., 1981), 513-536, Proc. Sympos. Pure Math. 40, Amer. Math. Soc., Providence, RI, 1983.

SV00 A. Suslin and V. Voevodsky, Bloch-Kato conjecture and motivic cohomology with finite coefficients, in The arithmetic and geometry of algebraic cycles (Banff, AB, 1998), 117-189, NATO Sci. Ser. C Math. Phys. Sci. 548, Kluwer Acad. Publ., Dordrecht, 2000.

Tra70 C. Traverso, Seminormality and Picard group, Ann. Scuola Norm. Sup. Pisa (3) 24 (1970), $585-595$. 


\section{Annette Huber And Clemens Jörder}

Voe96 V. Voevodsky, Homology of schemes, Selecta Math. (N.S.) 2 (1996), no. 1, 111-153.

Annette Huber annette.huber@math.uni-freiburg.de

Math. Institut, Universität Freiburg, Eckerstr. 1, 79104 Freiburg, Germany.

Clemens Jörder c.joerder@web.de

Math. Institut, Universität Freiburg, Eckerstr. 1, 79104 Freiburg, Germany. 